\title{
Stepwise Acid-Promoted Double-Michael Process: an Alternative to Diels-Alder Cycloadditions for Hindered Silyoxydiene-Dienophile Pairs
}

\author{
Michael E. Jung* and David G. Ho \\ Department of Chemistry and Biochemistry, University of California, Los Angeles, CA 90095-1569 \\ jung@chem.ucla.edu
}

Supporting Information

\section{Experimental Section}

General Methods. All reactions were carried out in flame-dried flasks under argon atmosphere unless otherwise specified. Tetrahydrofuran (THF) and diethyl ether were purified by distillation from sodium benzophenone ketyl, methanol distilled from magnesium turnings, and dichloromethane, benzene, toluene, triethylamine, pyridine, diisopropylamine, and hexamethylphosphoramide (HMPA) were distilled from calcium hydride. All dienes and dienophiles were prepared accordingly to the literature, unless otherwise stated. All other reagents were reagent grade and were purified where necessary. Reactions were monitored by thin-layer chromatography (TLC) using $0.2 \mathrm{~mm}$ Merck precoated silica gel $60-\mathrm{F}_{254}$ on aluminum sheet (using vanillin stains where necessary) and gas chromatography (GC) using a Hewlett Packard 6890 series instrument. Proton and carbon NMR spectra were recorded on a Bruker ARX400 $400 \mathrm{MHz}$, ARX500 $500 \mathrm{MHz}$, Avance500 $500 \mathrm{MHz}$, or Avance600 $600 \mathrm{MHz}$ spectrometer. ${ }^{1} \mathrm{H}$ and ${ }^{13} \mathrm{C}$ chemical shifts are reported as $\delta$ values (ppm) relative to TMS.

2-[(1,1-Dimethylethyl)dimethylsilyloxy]-4-methyl-1,3-pentadiene (1). To a solution of mesityl oxide (8.73 g, $0.89 \mathrm{~mol})$ and dry $\mathrm{Et}_{3} \mathrm{~N}(22.51 \mathrm{~g}, 0.22 \mathrm{~mol})$ in THF (50 mL) cooled to $0{ }^{\circ} \mathrm{C}$ was slowly added tert-Butyldimethylsilyl trifluoromethanesulfonate $(28.23 \mathrm{~g}, 0.11$ mol) over a $10 \mathrm{~min}$ period and allowed to stir for $3 \mathrm{~h}$. The mixture was warmed to $22{ }^{\circ} \mathrm{C}$ stirred for an additional $2 \mathrm{~h}$, then extracted with pentane $(100 \mathrm{~mL})$, washed with sat. $\mathrm{NaHCO}_{3}(2 \times 100 \mathrm{~mL})$, brine $(3 \times 100 \mathrm{~mL})$, and dried over $\mathrm{MgSO}_{4}$. It was then purified by distillation (80 ${ }^{\circ} \mathrm{C} / 10 \mathrm{~mm} \mathrm{Hg}$ ) to obtain 1 (17.39 g, 92\%; 92:8 mixture of isomers): ${ }^{1} \mathrm{H}$ NMR (500 MHz, $\left.\mathrm{CDCl}_{3}\right)$ $\delta: 5.56(1 \mathrm{H}, \mathrm{s}), 4.30(1 \mathrm{H}, \mathrm{s}), 4.16(1 \mathrm{H}, \mathrm{s}), 1.90(3 \mathrm{H}, \mathrm{s}), 1.77$ (3H, s), 0.95 (9H, s), 0.17 (6H, s); ${ }^{13} \mathrm{C}$ NMR (125 MHz, $\left.\mathrm{CDCl}_{3}\right)$ $\delta: 155.5,136.6,122.9,94.7$, 26.9, 25.7, 19.7, 18.2, -4.6.

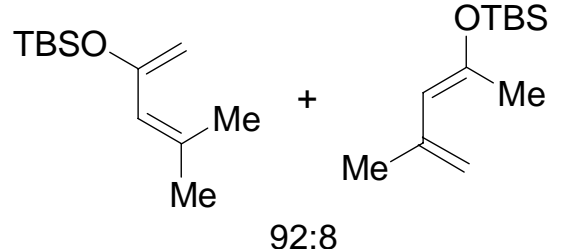


(Method A) General Procedure for Triflimide Promoted Cycloaddition. The enone (1 mol equiv.) and the diene (1.1 mol equiv.) were dissolved in dichloromethane (5 mL) and the mixture was cooled to $-78{ }^{\circ} \mathrm{C}$ (acetone/dry-ice). Triflimide $\left(\mathrm{Tf}_{2} \mathrm{NH}, 0.02\right.$ eq. of a $1 \mathrm{M}$ solution in dichloromethane) was added and the mixture was allowed to stir until complete disappearance of the enone as monitored by GC and TLC. Then trimethylaluminum $(0.1$ eq. of a $2 \mathrm{M}$ solution in toluene) was added and the mixture was warmed and kept at $-5{ }^{\circ} \mathrm{C}$ (refrigerator) over the required time to complete the cyclization as shown by GC monitoring. The reaction was quenched with pyridine $(1 \mathrm{~mL})$ and the mixture was filtered through a small pad of silica gel.

(Method B) General Procedure for Lewis Acid Catalyzed Cycloaddition. The enone (1 mol equiv.) was dissolved in a solution of toluene $(3 \mathrm{~mL})$ and dichloromethane $(1.5 \mathrm{~mL})$ and the mixture was cooled to $-9{ }^{\circ} \mathrm{C}$ (brine/ice). Trimethylaluminum (0.1 equiv. of a $2.0 \mathrm{M}$ solution in toluene) was slowly added to the solution and it was allowed to stir for $10 \mathrm{~min}$, followed by the slow addition of aluminum bromide ( 0.5 equiv. of a $1.0 \mathrm{M}$ solution in dibromomethane). The mixture was stirred for additional $15 \mathrm{~min}$ and the neat diene (1.0 equiv.) was added slowly to the mixture. The reaction was kept refrigerated at $-5{ }^{\circ} \mathrm{C}$ for 24 $\mathrm{h}$, then an additional 0.5 equiv. (or otherwise stated) of diene was added slowly and the reaction stirred for another $24 \mathrm{~h}$. The solution was quenched with pyridine $(2 \mathrm{~mL})$ and the mixture warmed to $23{ }^{\circ} \mathrm{C}$. The suspension was filtered through a small pad of silica gel, and the solvent removed in vacuo.

( $\pm(4 a S, 8 a S)-6-[(1,1-D i m e t h y l e t h y l) d i m e t h y l s i l y l] o x y-8,8,8 a-t r i m e t h y l-3,4,4 a, 5,8,8 a-$ hexahydronaphthalen-1(2H)-one (14). To a solution of enone $12(0.11 \mathrm{~g}, 1.00 \mathrm{mmol})$ and the diene $1(0.23 \mathrm{~g}, 1.1 \mathrm{mmol})$ in $5 \mathrm{~mL}$ dry dichloromethane cooled to $-78{ }^{\circ} \mathrm{C}$ was added triflimide $(0.05 \mathrm{mmol})$ and the mixture was stirred for $20 \mathrm{~min}$. Then trimethylaluminum $(0.05 \mathrm{~mL}$ of a $2.0 \mathrm{M}$ solution in toluene, $0.10 \mathrm{mmol})$ was added and the mixture was treated as described in the general procedure (5 days for completion). Column chromatography over silica gel (95:5 hexanes:diethyl ether with $1 \%$ triethylamine) yielded the desired compound $14(0.28 \mathrm{~g}, 0.86 \mathrm{mmol}, 86 \%):{ }^{1} \mathrm{H}$ NMR $\left(500 \mathrm{MHz}, \mathrm{CDCl}_{3}\right) \delta: 4.63$ $(1 \mathrm{H}, \mathrm{s}), 2.63(1 \mathrm{H}, \mathrm{dd}, J=15.5,7.1 \mathrm{~Hz}), 2.28(1 \mathrm{H}, \mathrm{m}), 2.22-2.18(1 \mathrm{H}, \mathrm{m}), 2.11(1 \mathrm{H}, \mathrm{ddd}, J$ = 8.5, 7.2, $1.4 \mathrm{~Hz}), 2.0-1.82(4 \mathrm{H}, \mathrm{m}), 1.68(1 \mathrm{H}, \mathrm{m}), 1.24(3 \mathrm{H}, \mathrm{s}), 1.15(3 \mathrm{H}, \mathrm{s}), 0.90(3 \mathrm{H}, \mathrm{s})$, 0.89 (9H, s), 0.11 (3H, s), 0.10 (3H, s); ${ }^{13} \mathrm{C}$ NMR $\left(125 \mathrm{MHz}, \mathrm{CDCl}_{3}\right) \delta: 213.9,144.9$, 115.3, 52.4, 41.2, 39.3, 36.6, 32.0, 28.4, 27.4, 26.1, 25.5, 23.0, 20.2, 17.8, -4.5, -4.6.

Method B: The dienophile $12(0.22 \mathrm{~g}, 2.0 \mathrm{mmol})$, trimethylaluminum $(0.1 \mathrm{~mL}$ of a $2.0 \mathrm{M}$ solution in toluene, $0.2 \mathrm{mmol})$, aluminum bromide $(1.0 \mathrm{~mL}$ of a $1.0 \mathrm{M}$ solution in dibromomethane, $1.0 \mathrm{mmol})$, and the diene $\mathbf{1}(0.64 \mathrm{~g}, 3.0 \mathrm{mmol})$ were treated as described in the general procedure B (2h). Column chromatography over silica gel (95:1 hexanes:diethyl ether with $2 \%$ triethylamine) yielded the desired compound 14 (0.23 g, $0.72 \mathrm{mmol}, 72 \%)$.

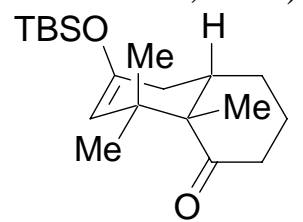


( \pm (4aS,8aS)-6-[(1,1-Dimethylethyl)dimethylsilyl]oxy-8,8,8a-trimethyl-3,4,4a,7,8,8ahexahydronaphthalen-1(2H)-one (16). The enone $12(0.11 \mathrm{~g}, 1.00 \mathrm{mmol})$ and the diene 1 $(0.23 \mathrm{~g}, 1.1 \mathrm{mmol})$ in $5 \mathrm{~mL}$ dry dichloromethane cooled to $-78{ }^{\circ} \mathrm{C}$ was added triflimide $(0.05 \mathrm{mmol})$ and the mixture was stirred for $20 \mathrm{~min}$. Then trimethylaluminum $(0.05 \mathrm{~mL}$ of a $2.0 \mathrm{M}$ solution in toluene, $0.10 \mathrm{mmol})$ and aluminum bromide $(0.5 \mathrm{~mL}$ of a $1.0 \mathrm{M}$ solution in dibromomethane, $0.50 \mathrm{mmol}$ ) were added and the mixture was treated as described in the general procedure (15 h for completion). Column chromatography over silica gel (95:5 hexanes:diethyl ether with $1 \%$ triethylamine) yielded isomer 16 (0.26 g, $0.82 \mathrm{mmol}, 82 \%):{ }^{1} \mathrm{H}$ NMR (500 MHz, $\left.\mathrm{CDCl}_{3}\right) \delta: 4.50(1 \mathrm{H}, \mathrm{s}), 2.59(1 \mathrm{H}, \mathrm{bd}, J=15.7 \mathrm{~Hz}$ ), $2.58(1 \mathrm{H}, \mathrm{m}) 2.51(1 \mathrm{H}, \mathrm{ddd}, J=14.5,13.3,6.9 \mathrm{~Hz}), 2.09(1 \mathrm{H}, \mathrm{bd}, J=15.4 \mathrm{~Hz}), 2.05(1 \mathrm{H}$, bd, $J=15.5 \mathrm{~Hz}$ ), 1.81-1.72 (2H, m), 1.55 (2H, m), 1.18 (3H, s), 1.17 (3H, s), 0.88 (9H, s), 0.84 (3H, s), 0.07 (3H, s), 0.06 (3H, s); ${ }^{13} \mathrm{C}$ NMR (125 MHz, $\left.\mathrm{CDCl}_{3}\right) \delta: 214.2,152.0$, 107.4, 51.5, 42.0, 41.1, 39.7, 35.6, 27.2, 25.6, 24.9, 24.8, 23.4, 17.8, 17.1, -4.4, -4.5.

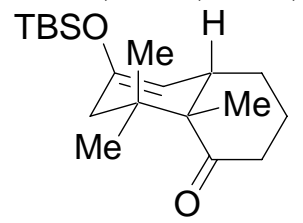

( \pm (4aS,8aS)-8,8,8a-Trimethylhexahydronaphthalene-1,6(2H,5H)-dione (15). The silyl enol ether $14(0.11 \mathrm{~g}, 0.34 \mathrm{mmol})$ was dissolved in $6 \mathrm{~mL}$ of 2:1 $p$-dioxane/water mixture at $23{ }^{\circ} \mathrm{C}$. One drop of conc. $\mathrm{HCl}$ was added to the mixture and the reaction was stirred overnight $(15 \mathrm{~h})$. Sodium bicarbonate was added to quench the reaction, followed by extraction with diethyl ether $(3 \times 10 \mathrm{~mL})$ and drying over anhydrous magnesium sulfate. Then the solution was filtered through a small pad of silica gel and the solvent removed in vacuo to yield the dione 15 (0.07 g, $0.32 \mathrm{mmol}, 95 \%):{ }^{1} \mathrm{H}$ NMR $\left(500 \mathrm{MHz}, \mathrm{C}_{6} \mathrm{D}_{6}\right) \delta: 3.21$ $(1 \mathrm{H}, \mathrm{d}, J=14.0 \mathrm{~Hz}), 2.83(1 \mathrm{H}, \mathrm{dd}, J=13.6,7.7 \mathrm{~Hz}), 2.51(1 \mathrm{H}, \mathrm{m}), 2.30-2.16(3 \mathrm{H}, \mathrm{m}), 2.10$ (1H, dddd, $J=14.6,2.5,2.5,2.5 \mathrm{~Hz}), 2.0-1.89(3 \mathrm{H}, \mathrm{m}), 1.40(1 \mathrm{H}, \mathrm{m}), 1.39$ (3H, s), 1.19 (3H, s), 0.80 (3H, s); ${ }^{13} \mathrm{C}$ NMR (125 MHz, $\left.\mathrm{CDCl}_{3}\right) \delta: 214.6,211.2,66.9,53.3,52.3,43.6$, 43.3, 40.1, 39.2, 25.5, 25.0, 22.8, 18.7.

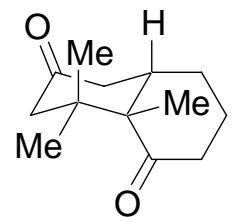

( \pm 1-\{3-[(1,1-Dimethylethyl)dimethylsilyloxy]-2-methylcyclohex-2-enyl\}-4-methylpent3-en-2-one (13). To a solution of enone $12(0.11 \mathrm{~g}, 1.00 \mathrm{mmol})$ and the diene $\mathbf{1}(0.23 \mathrm{~g}, 1.1$ $\mathrm{mmol})$ in $5 \mathrm{~mL}$ dry dichloromethane cooled to $-78{ }^{\circ} \mathrm{C}$ was added triflimide $(0.05 \mathrm{mmol})$ and the mixture was stirred for $20 \mathrm{~min}$. The reaction was quenched with $\mathrm{Et}_{3} \mathrm{NH}(1 \mathrm{~mL})$ and the mixture was filtered through a small pad of silical gel. Column chromatography over silica gel (9:1 hexanes:diethyl ether with $1 \%$ triethylamine) yielded the desired compound 13 (0.31 g, $0.95 \mathrm{mmol}, 95 \%):{ }^{1} \mathrm{H}$ NMR (500 MHz, $\left.\mathrm{CDCl}_{3}\right) \delta$ : $6.06(1 \mathrm{H}, \mathrm{s}), 2.59(2 \mathrm{H}, \mathrm{m})$, $2.56(1 \mathrm{H}, \mathrm{dd}, J=16.2,10.9 \mathrm{~Hz}), 2.13(3 \mathrm{H}, \mathrm{s}), 1.99(2 \mathrm{H}, \mathrm{m}), 1.87$ (3H, s), 1.16-1.57 (3H, m), 1.56 (3H, s) 0.92 (9H, s), 0.1 (6H, s), 0.07 (3H, s), 0.06 (3H, s); ${ }^{13} \mathrm{C}$ NMR $(125 \mathrm{MHz}$, $\left.\mathrm{CDCl}_{3}\right) \delta$ : 201.0, 154.7, 144.5, 124.0, 113.8, 48.1, 35.4, 30.3, 27.7, 27.5, 25.7, 20.6, 19.8, 18.1, 14.5, -3.9, -4.0 . 
Method B: The enone $12(0.11 \mathrm{~g}, 1.00 \mathrm{mmol})$ and trimethylaluminum $(0.01 \mathrm{~mL}$ of a $2.0 \mathrm{M}$ solution in toluene, $0.1 \mathrm{mmol}$ ) were dissolved in $5 \mathrm{~mL}$ dry dichloromethane cooled to -78 ${ }^{\circ} \mathrm{C}$ and the mixture was stirred for $15 \mathrm{~min}$. Aluminum bromide $(0.5 \mathrm{~mL}$ of a $1.0 \mathrm{M}$ solution in dibromomethane, $0.5 \mathrm{mmol}$ ) was added and the mixture was stirred for $15 \mathrm{~min}$. The diene $1(0.32 \mathrm{~g}, 1.5 \mathrm{mmol})$ was then added. After stirring $2 \mathrm{~h}$, the mixture was quenched with pyridine $(1 \mathrm{~mL})$ and filtered through a small pad of silical gel. Column chromatography of the residue over silica gel (9:1 hexanes:diethyl ether with 1\% triethylamine) yielded the desired compound 13 (0.29g, $0.92 \mathrm{mmol}, 92 \%)$.<smiles>CCOC1=C(O)C(CC(=O)C=C(C)C)CCC1</smiles>

1-\{3-[(1,1-Dimethylethyl)dimethylsilyloxy]-1-methylcyclohex-2-enyl\}-4-methylpent-3en-2-one (7). To a solution of enone $6(0.11 \mathrm{~g}, 1.00 \mathrm{mmol})$ and the diene $1(0.23 \mathrm{~g}, 1.1$ $\mathrm{mmol})$ in $5 \mathrm{~mL}$ dry dichloromethane cooled to $-78{ }^{\circ} \mathrm{C}$ was added triflimide $(0.05 \mathrm{mmol})$ and the mixture was stirred for $2 \mathrm{~h}$. The the reaction was quench with $\mathrm{Et}_{3} \mathrm{NH}(1 \mathrm{~mL})$ and the mixture was filtered through a small pad of silical gel. Column chromatography over silica gel (9:1 hexanes:diethyl ether with $1 \%$ triethylamine) yielded the desired compound 7 (0.29g, $0.89 \mathrm{mmol}, 89 \%):{ }^{1} \mathrm{H}$ NMR (500 MHz, $\left.\mathrm{CDCl}_{3}\right) \delta: 6.05(1 \mathrm{H}, \mathrm{s}), 4.75$ (1H, s), 2.38 $(1 \mathrm{H}, \mathrm{d}, J=13.1 \mathrm{~Hz}), 2.31(1 \mathrm{H}, \mathrm{d}, J=13.1 \mathrm{~Hz}), 2.11(3 \mathrm{H}, \mathrm{s}), 1.94(2 \mathrm{H}, \mathrm{m}), 1.85$ (3H, s), $1.67(2 \mathrm{H}, \mathrm{m}), 1.56(1 \mathrm{H}, \mathrm{m}) 1.36(1 \mathrm{H}, \mathrm{m}), 1.07(3 \mathrm{H}, \mathrm{s}), 0.90(9 \mathrm{H}, \mathrm{s}), 0.11(3 \mathrm{H}, \mathrm{s}), 0.10(3 \mathrm{H}$, s); ${ }^{13} \mathrm{C}$ NMR (125 MHz, $\left.\mathrm{CDCl}_{3}\right) \delta: 200.6,153.9,149.9,125.8,113.6,56.0,35.4,35.2$, 29.6, 28.2, 27.5, 25.6, 20.5, 19.3, 17.9, -4.5, -4.6.

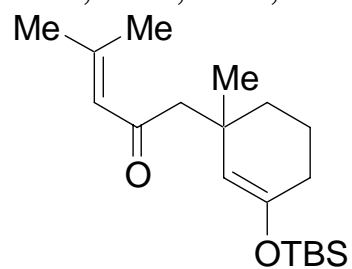

\section{9-[(1,1-Dimethylethyl)dimethylsilyloxy]-4a,4b-dimethyl-2,3,4a,4b,5,6,7,8,10,10a-} decahydro-1H-phenanthren-4-one (24). The dienophile 12 (0.11 g, $1.0 \mathrm{mmol})$, trimethylaluminum $(0.05 \mathrm{~mL}$ of a $2.0 \mathrm{M}$ solution in toluene, $0.1 \mathrm{mmol})$, aluminum bromide ( $0.5 \mathrm{~mL}$ of a $1.0 \mathrm{M}$ solution in dibromomethane, $0.5 \mathrm{mmol})$, and the diene $23(0.50 \mathrm{~g}, 2.0$ mmol) were treated as described in the general procedure B (48h). Column chromatography over silica gel (95:1 hexanes:diethyl ether with $2 \%$ triethylamine) yielded the desired compound $24(0.26 \mathrm{~g}, 0.71 \mathrm{mmol}, 71 \%):{ }^{1} \mathrm{H}$ NMR $\left(500 \mathrm{MHz}, \mathrm{CDCl}_{3}\right) \delta: 2.82$ (1H, dddd, $J=14.1,2.2,2.2,2.2 \mathrm{~Hz}), 2.55(1 \mathrm{H}, \mathrm{m}), 2.45-2.32(2 \mathrm{H}, \mathrm{m}), 2.09(1 \mathrm{H}, \mathrm{m}), 1.98$ (3H, m), 1.9-1.4 (7H, m), 1.21 (3H, s), 1.20 (3H, s), 0.94 (9H, s), 0.12 (3H, s), 0.11 (3H, s); ${ }^{13} \mathrm{C}$ NMR $\left(125 \mathrm{MHz}, \mathrm{CDCl}_{3}\right) \delta$ : 215.6, 138.1, 118.8, 52.6, 41.8, 39.8, 39.4, 35.1, 33.0, 27.4, 26.5, 25.8, 23.5, 22.2, 22.0, 21.1, 18.1, -4.0, -4.1. 


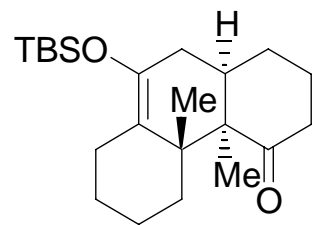

4a,4b-Dimethyldecahydrophenanthrene-4,9-dione (27). The silyl enol ether 24 (0.13 g, $0.35 \mathrm{mmol})$ was dissolved in methanol $(6 \mathrm{~mL})$ at $23{ }^{\circ} \mathrm{C} . \quad p$-Toluenesulfonic acid $(0.07 \mathrm{~g}$, $0.35 \mathrm{mmol})$ was added to the mixture and the reaction was stirred overnight $(18 \mathrm{~h})$. A spatula tip of solid sodium bicarbonate was added to quench the reaction and the solvent was removed in vacuo. The crude was redissolved in dichloromethane $(5 \mathrm{~mL})$ and dried over anhydrous magnesium sulfate. It was then filtered through a small pad of silica gel with 1:1 $\mathrm{CH}_{2} \mathrm{Cl}_{2}$ :hexanes, and the solvent was removed in vacuo to yield the dione 27 (0.08 g, $0.31 \mathrm{mmol}, 90 \%):{ }^{1} \mathrm{H} \mathrm{NMR}\left(500 \mathrm{MHz}, \mathrm{CDCl}_{3}\right) \delta: 3.14(1 \mathrm{H}, \mathrm{m}), 2.93(1 \mathrm{H}$, ddd, $J=13.3$, 8.1, 8.1 Hz), $2.63(1 \mathrm{H}, \mathrm{m}), 2.45-1.25(8 \mathrm{H}, \mathrm{m}), 1.60-1.35(6 \mathrm{H}, \mathrm{m}), 1.42(3 \mathrm{H}, \mathrm{s}), 1.32$ (3H, s), $1.13(1 \mathrm{H}, \mathrm{m})$; ${ }^{13} \mathrm{C} \mathrm{NMR}\left(125 \mathrm{MHz}, \mathrm{CDCl}_{3}\right) \delta: 215.0,212.6,54.2,50.9,43.9,43.7,43.2$, 39.8, 30.8, 25.6, 24.1, 22.7, 21.6, 21.0, 18.1, 14.8.<smiles>[M][C@]12CCCCC1C(=O)C[C@H]1CCCC(=O)[C@@]12O</smiles>

1-\{9-[(1,1-Dimethylethyl)dimethylsilyloxy]-4b,10a-dimethyl-1,2,3,4,4a,4b,5,6,7,8,10, 10a-dodecahydrophenanthren-4a-yl\}-ethanone (29,30). The dienophile 28 (0.17 g, 1.25 mmol), trimethylaluminum $(0.06 \mathrm{~mL}$ of a $2.0 \mathrm{M}$ solution in toluene, $0.1 \mathrm{mmol})$, aluminum bromide $(0.6 \mathrm{~mL}$ of a $1.0 \mathrm{M}$ solution in dibromomethane, $0.6 \mathrm{mmol})$, and the diene 23 (0.47 g, $1.9 \mathrm{mmol})$ were treated as described in the general procedure B (48h). Column chromatography over silica gel (95:1 hexanes:diethyl ether with $2 \%$ triethylamine) yielded the desired mixture 6.4:1 compound $29+30$ (0.43 g, $1.1 \mathrm{mmol}, 88 \%):{ }^{1} \mathrm{H}$ NMR (500 MHz, $\mathrm{CDCl}_{3}$ ) $\delta$ (major): 2.91 (1H, bd, $\left.J=13.9 \mathrm{~Hz}\right), 2.68(1 \mathrm{H}, \mathrm{bd}, J=16.9 \mathrm{~Hz}), 2.16(3 \mathrm{H}, \mathrm{s}), 2.08$ $(1 \mathrm{H}, \mathrm{m}), 1.96$ (1H, ddd, $J=14.0,4.9,4.9 \mathrm{~Hz}), 1.84-1.40(11 \mathrm{H}, \mathrm{m}), 1.40(3 \mathrm{H}, \mathrm{s}), 1.20(1 \mathrm{H}$, m), 1.10 (3H, s), $1.01(2 \mathrm{H}, \mathrm{m}), 0.93(9 \mathrm{H}, \mathrm{s}), 0.11(3 \mathrm{H}, \mathrm{s}), 0.10(3 \mathrm{H}, \mathrm{s}) ;{ }^{13} \mathrm{C}$ NMR (125

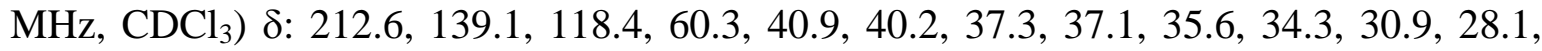
27.5, 26.6, 25.8, 23.5, 22.2, 22.0, 20.9, 20.4, 18.1, -3.7, -3.8.

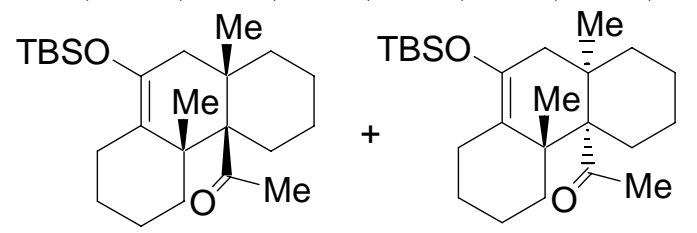

6.4:1

Methy 7-[(1,1-dimethylethyl)dimethylsilyloxy]-5,5,8a-trimethyl-4-oxo-1,2,3,4,4a,5,8,8a -octahydronaphthaleneacetate (22). To a solution of enone 21 (1.47 g, $8.06 \mathrm{mmol})$ and the diene 1 ( $2.05 \mathrm{~g}, 9.67 \mathrm{mmol})$ in $70 \mathrm{~mL}$ dry dichloromethane cooled to $-78{ }^{\circ} \mathrm{C}$ was added triflimide $(0.4 \mathrm{mmol})$ and the mixture was stirred for $2 \mathrm{~h}$. After the mixtures was then 
slowly warm to $0{ }^{\circ} \mathrm{C}$ over 10 min. periods, quenched with dry triethylamine $(2 \mathrm{~mL})$ and filtered through a small pad of silica. Column chromatography over silica gel (9:1 hexanes:diethyl ether with $2 \%$ triethylamine) yielded the desired compound 22 (2.73 g, $6.93 \mathrm{mmol}, 86 \%):{ }^{1} \mathrm{H}$ NMR (500 MHz, $\left.\mathrm{CDCl}_{3}\right) \delta: 4.61(1 \mathrm{H}, \mathrm{s}), 3.68(3 \mathrm{H}, \mathrm{s}), 2.65(1 \mathrm{H}, \mathrm{m})$, $2.51(4 \mathrm{H}, \mathrm{m}), 2.15-1.96(3 \mathrm{H}, \mathrm{m}), 1.85(1 \mathrm{H}, \mathrm{ddd}, J=8.1,2.1,2.1 \mathrm{~Hz}), 1.63(1 \mathrm{H}, \mathrm{m}), 1.10$ (3H, s), 1.09 (3H, s), 0.92 (9H, s), 0.16 (3H, s), 0.15 (3H, s); ${ }^{13} \mathrm{C}$ NMR $\left(125 \mathrm{MHz}, \mathrm{CDCl}_{3}\right.$ ) $\delta:$ 213.1, 173.2, 145.4, 113.2, 65.2, 51.5, 39.8, 39.1, 39.0, 35.8, 34.6, 33.7, 31.7, 29.5, 26.4, 25.8, 25.6, 23.2, 17.9, -4.6.

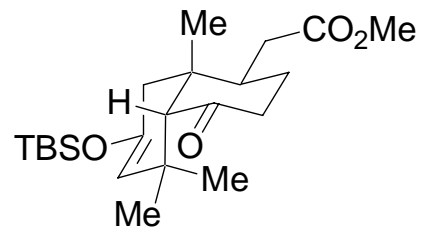

1-\{2-(1-[(1,1-Dimethylethyl)dimethylsilyloxy]-ethylidene)-1-methyl-cyclopentyl\}-4methyl-pent-3-en-2-one (20ab) The enone $\mathbf{1 7}(0.12 \mathrm{~g}, 1.00 \mathrm{mmol})$ and the diene $\mathbf{1}(0.23 \mathrm{~g}$, $1.1 \mathrm{mmol})$ in $5 \mathrm{~mL}$ dry $\mathrm{CH}_{2} \mathrm{Cl}_{2}$ at $-78{ }^{\circ} \mathrm{C}$ was added $\mathrm{Tf}_{2} \mathrm{NH}(0.05 \mathrm{mmol})$ and stirred for 20 min. Then quench with $\mathrm{Et}_{3} \mathrm{NH}(1 \mathrm{~mL})$. Column chromatography over silica gel (9:1 hexanes:diethyl ether with $1 \%$ triethylamine) yielded $8: 1$ isomers $20 \mathrm{ab}(0.30 \mathrm{~g}, 0.90 \mathrm{mmol}$, 90\%): ${ }^{1} \mathrm{H}$ NMR (500 MHz, $\left.\mathrm{CDCl}_{3}\right) \delta$ (major): $6.17(1 \mathrm{H}, \mathrm{s}), 2.80(1 \mathrm{H}, \mathrm{d}, J=12.8 \mathrm{~Hz}), 2.64$ (1H, d, $J=12.8 \mathrm{~Hz}$ ), 2.49-2.35 (2H, m), 2.28 (2H, brt, $J=7.5 \mathrm{~Hz}), 2.09$ (3H, s), 1.84 (3H, s), $1.80(3 \mathrm{H}, \mathrm{s}), 1.67(2 \mathrm{H}, \mathrm{m}), 1.49(3 \mathrm{H}, \mathrm{s}), 0.87$ (9H, s), 0.07 (3H, s), 0.03 (3H, s); Partial ${ }^{1} \mathrm{H}$ NMR of minor $\left(400 \mathrm{MHz}, \mathrm{CDCl}_{3}\right) \delta($ minor): $6.08(1 \mathrm{H}, \mathrm{s}), 2.89(1 \mathrm{H}, \mathrm{d}, J=12.8 \mathrm{~Hz})$, $2.72(1 \mathrm{H}, \mathrm{d}, J=12.8 \mathrm{~Hz}), 1.83$ (3H, s), $1.57(3 \mathrm{H}, \mathrm{s}), 1.22$ (3H, s), 0.98 (9H, s), 0.20 (3H, s), 0.19 (3H, s); ${ }^{13} \mathrm{C}$ NMR $\left(125 \mathrm{MHz}, \mathrm{CDCl}_{3}\right.$ ) $\delta$ (major): 199.3, 153.2, 139.3, 133.2, 125.6, 75.5, 57.4, 52.3, 40.8, 35.7, 28.5, 27.4, 26.0, 21.4, 20.8, 20.4, 18.3, 16.2, -1.9, -2.8.

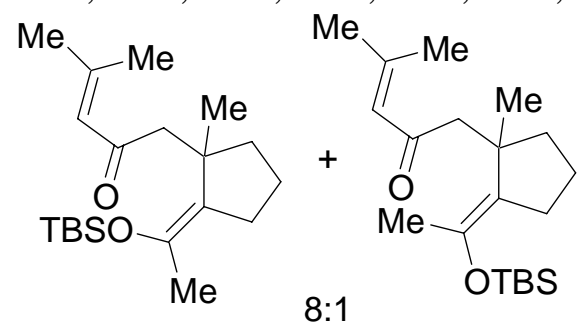

9-[(1,1-Dimethylethyl)dimethylsilyloxy]-4a,4b,8,8-tetramethyl-2,3,4a,4b,5,6,7,8,10,10adecahydro-1H-phenanthren-4-one (32) The dienophile 12 (0.05 g, $0.44 \mathrm{mmol})$, trimethylaluminum $(0.02 \mathrm{~mL}$ of a $2.0 \mathrm{M}$ solution in toluene, $0.04 \mathrm{mmol})$, aluminum bromide $(0.22 \mathrm{~mL}$ of a $1.0 \mathrm{M}$ solution in dibromomethane, $0.22 \mathrm{mmol})$, and the diene $\mathbf{3 1}$ $(0.17 \mathrm{~g}, 0.55 \mathrm{mmol})$ were treated as described in the general procedure $(8 \mathrm{~d})$. Column chromatography over silica gel (95:1 hexanes:diethyl ether with $2 \%$ triethylamine) yielded the desired compound $32(0.10 \mathrm{~g}, 0.27 \mathrm{mmol}, 61 \%):{ }^{1} \mathrm{H}$ NMR $\left(500 \mathrm{MHz}, \mathrm{CDCl}_{3}\right) \delta: 2.56$ (2H, m), 2.44 (1H, ddd, $J=15.4,13.2,6.0 \mathrm{~Hz}), 2.11$ (2H, dd, $J=17.5,4.4 \mathrm{~Hz}), 2.05$ (2H, m), $1.93(1 \mathrm{H}$, dddd, $J=12.2,3.4 \mathrm{~Hz}), 1.85(1 \mathrm{H}, \mathrm{m}), 1.78(2 \mathrm{H}, \mathrm{m}), 1.58-1.38(4 \mathrm{H}, \mathrm{m}), 1.37$ (3H, s), 1.29 (3H, s), 1.25 (1H, m), 1.22 (3H, s), 1.09 (3H, s),1.01 (9H, s), 0.25 (3H, s), 0.24 (3H, s); ${ }^{13} \mathrm{C}$ NMR $\left(125 \mathrm{MHz}, \mathrm{CDCl}_{3}\right) \delta: 216.0,143.5,124.3,55.1,43.6,42.3$ (2Cs), 35.8, 34.8, 33.6, 33.2, 30.3, 28.5, 28.4, 26.4, 25.8, 23.4, 18.6, 17.5, -2.0, -2.7. 


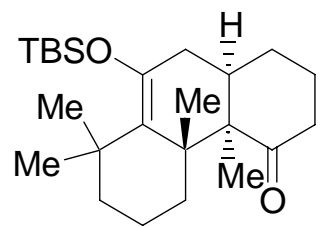

4a,4b,8,8-Tetramethyl-decahydro-phenanthrene-4,9-dione $(33,34)$. The silyl enol ether $32(0.05 \mathrm{~g}, 0.13 \mathrm{mmol})$ was dissolved in methanol $(6 \mathrm{~mL})$ at $23{ }^{\circ} \mathrm{C}$. p-Toluenesulfonic acid ( $0.04 \mathrm{~g}, 0.18 \mathrm{mmol})$ was added to the mixture and the reaction was stirred overnight (24 h). A spatula tip of solid sodium bicarbonate was added to quench the reaction and the solvent was removed in vacuo. The crude was redissolved in dichloromethane $(5 \mathrm{~mL})$ and dried over anhydrous magnesium sulfate. It was then filtered through a small pad of silica gel with 1:1 $\mathrm{CH}_{2} \mathrm{Cl}_{2}$ :hexanes, and the solvent was removed in vacuo to yield 8:2 isomers $33+$ 34 (0.03 g, $0.11 \mathrm{mmol}, 89 \%):{ }^{1} \mathrm{H}$ NMR (500 MHz, $\left.\mathrm{CDCl}_{3}\right) \delta$ (major): $2.70(1 \mathrm{H}, \mathrm{dd}, J=$ 14.1, $7.7 \mathrm{~Hz}), 2.52$ (1H, m), $2.39(1 \mathrm{H}, \mathrm{s}), 2.33(2 \mathrm{H}, \mathrm{m}), 2.27$ (1H, dd, $J=10.5,3.7 \mathrm{~Hz})$, 1.92 (2H, m), 1.80-1.61 (3H, m), 1.55 (2H, m), $1.45(3 \mathrm{H}, \mathrm{s}), 1.42(1 \mathrm{H}, \mathrm{m}), 1.27$ (3H, s), 1.10 (3H, s), 1.05 (1H, ddd, $J=13.3,4.0,4.0 \mathrm{~Hz}), 0.99(3 \mathrm{H}, \mathrm{s})$; Partial ${ }^{1} \mathrm{H}$ NMR of minor (500 MHz, $\left.\mathrm{CDCl}_{3}\right) \delta$ (minor): $2.73(1 \mathrm{H}, \mathrm{s}), 2.68-2.57(2 \mathrm{H}, \mathrm{m}), 2.45(1 \mathrm{H}, \mathrm{dd}, J=12.3,8.6$ Hz), 2.22 (1H, ddd, $J=8.0,1.6,1.6 \mathrm{~Hz}), 2.15$ (3H, m), 1.41 (3H, s), 1.32 (3H, s), 1.13 (3H, s), $0.91(3 \mathrm{H}, \mathrm{s}) ;{ }^{13} \mathrm{C}$ NMR (125 $\left.\mathrm{MHz}, \mathrm{CDCl}_{3}\right) \delta$ (major): 214.6, 211.2, 59.2, 55.5, 46.4, 46.1, 46.0, 42.6, 41.3, 34.8, 33.7, 32.7, 29.1, 23.1, 22.4, 22.3, 21.6, 17.7

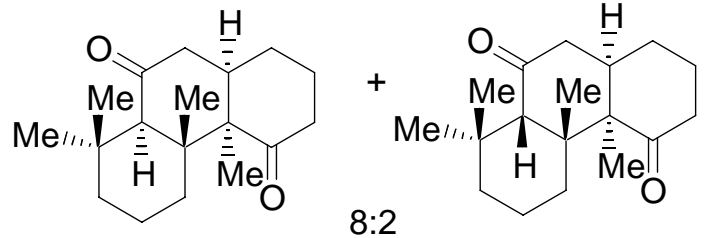



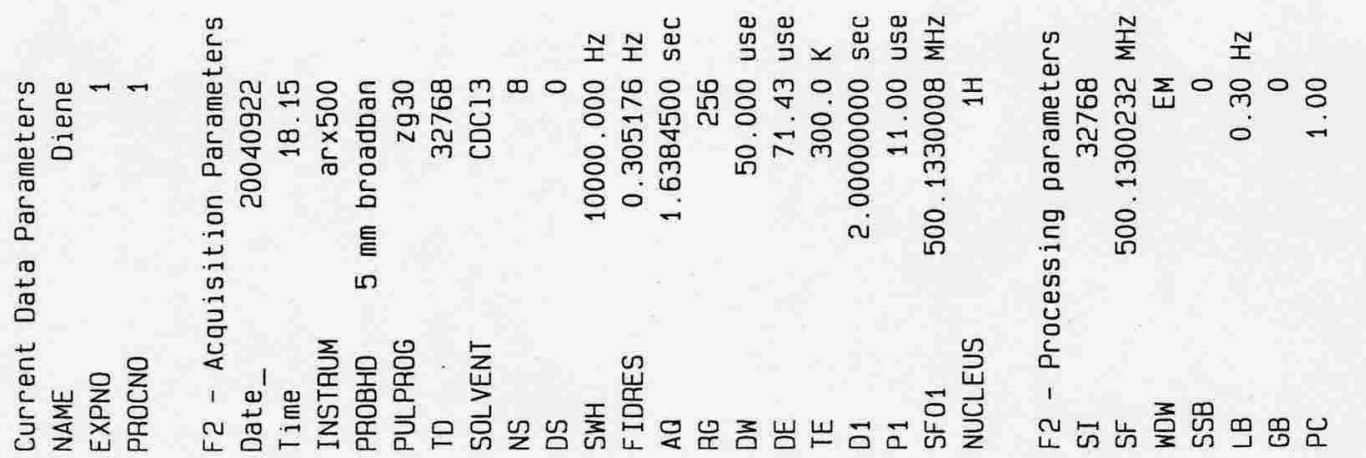

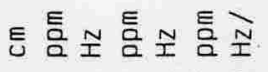

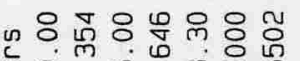

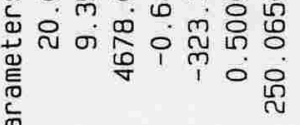
담 뭄

旁

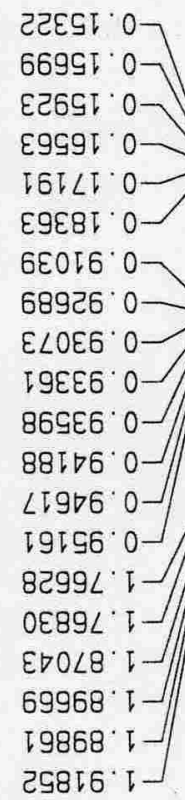

9द्ट9:

टEEOE' $\square$

ILLGG' 9 82099 9

98299. 9
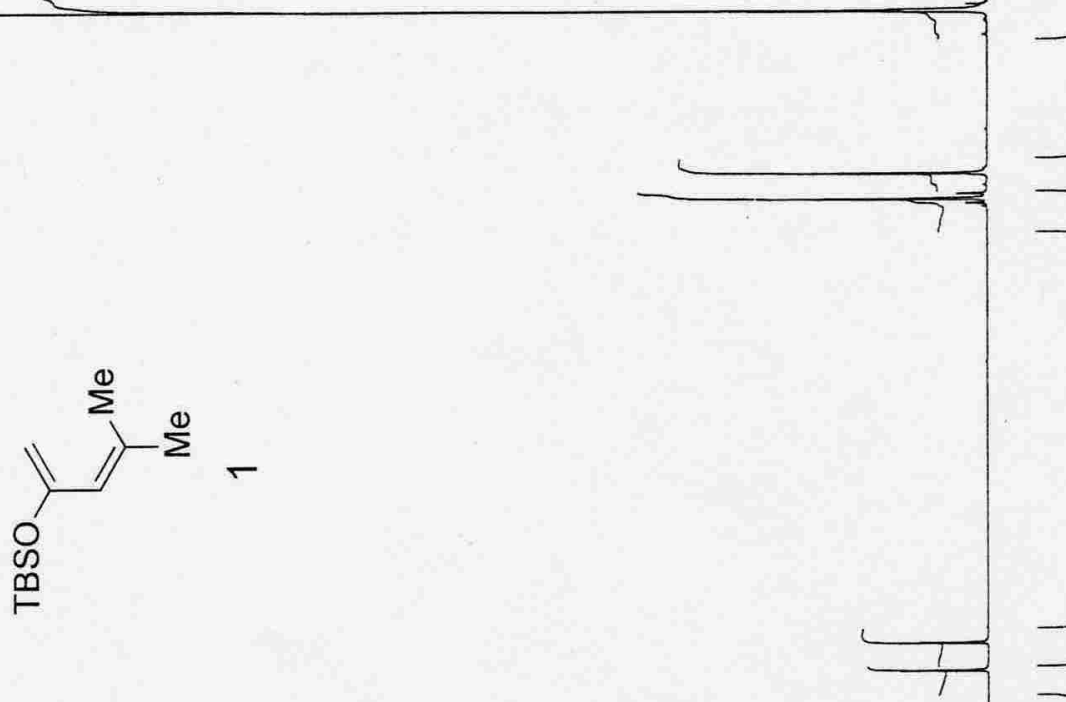

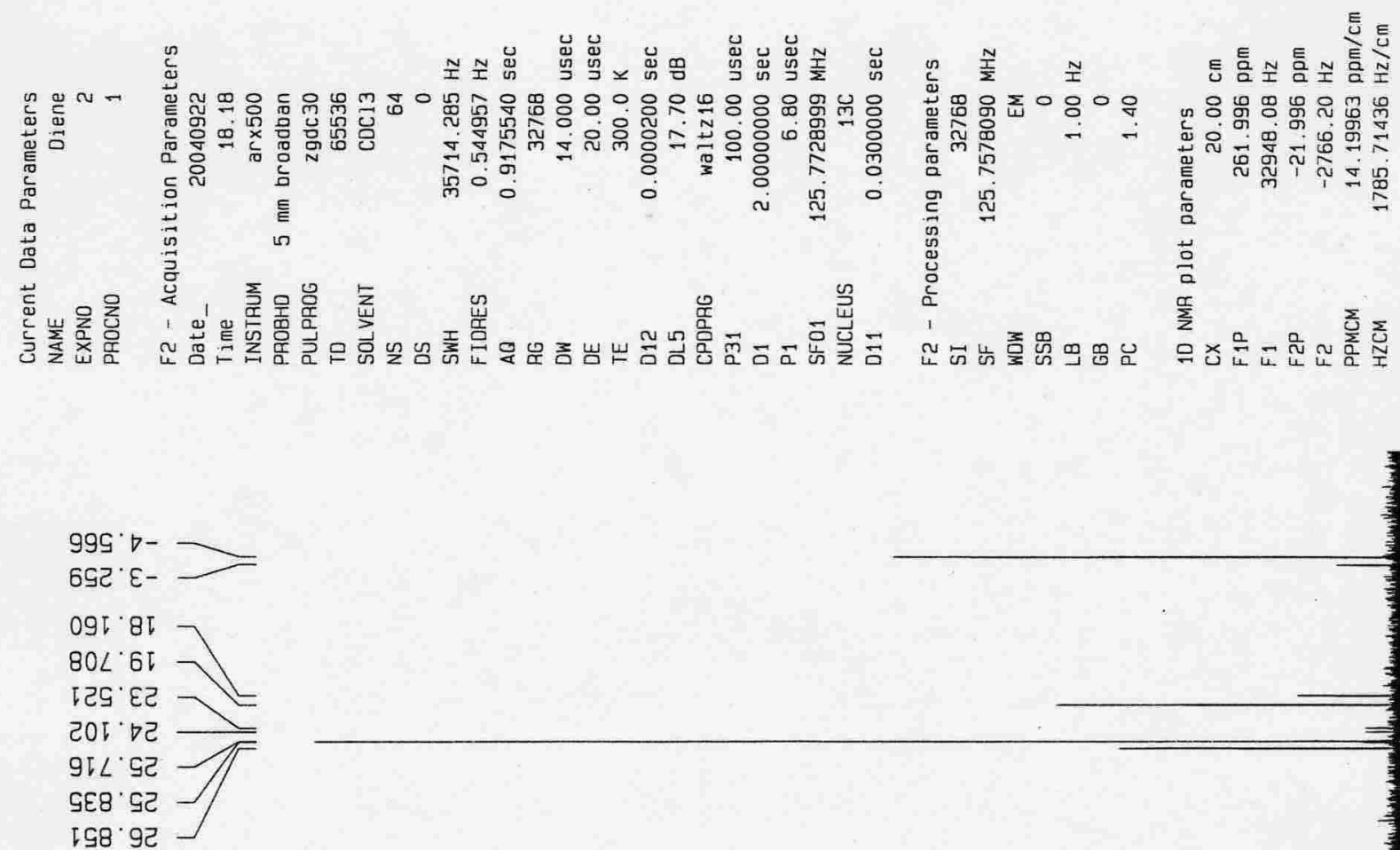

टE9 $9 L$

$988 \cdot 9 L$

项' $L L$

$9 \varepsilon \angle \triangleright \nabla 6$

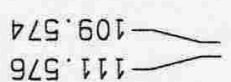

6टБ टटा-

$\angle L G \cdot 9 E I-$

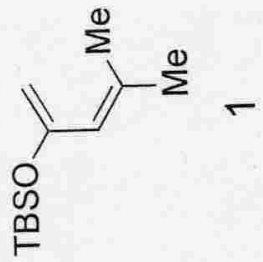

ટદ૬ ૬૬૬

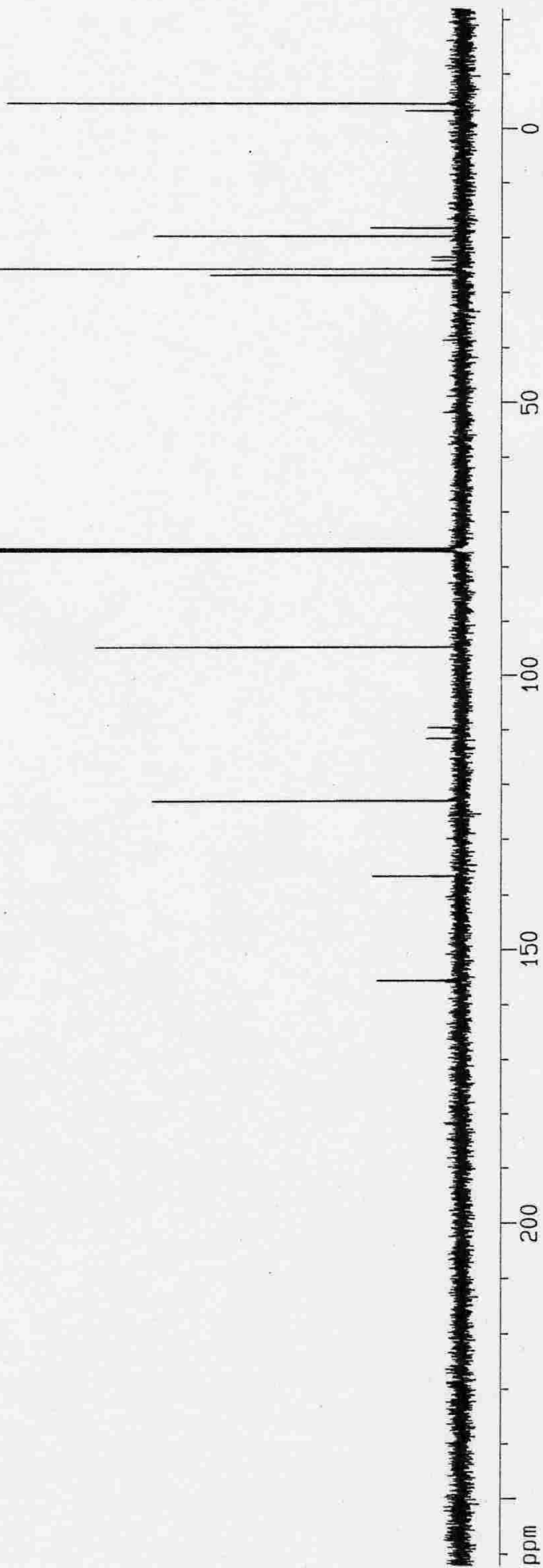




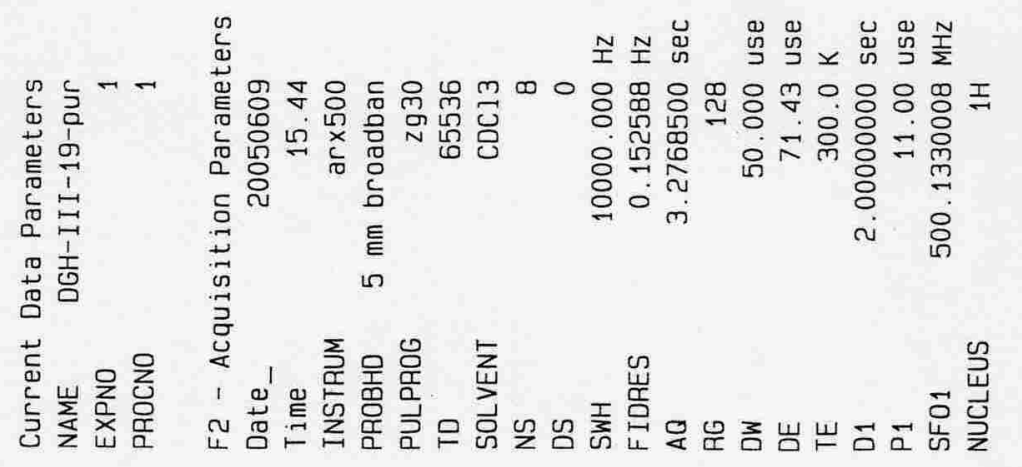

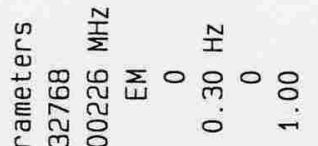

๕

담

c

트ㅁㅡㅡㅁ소음소 틈소

๑ : ㅇㅇㅇㅇㅇ

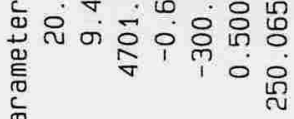

范

识

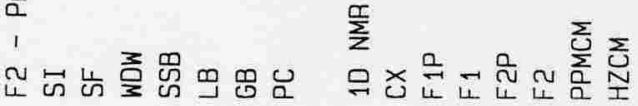

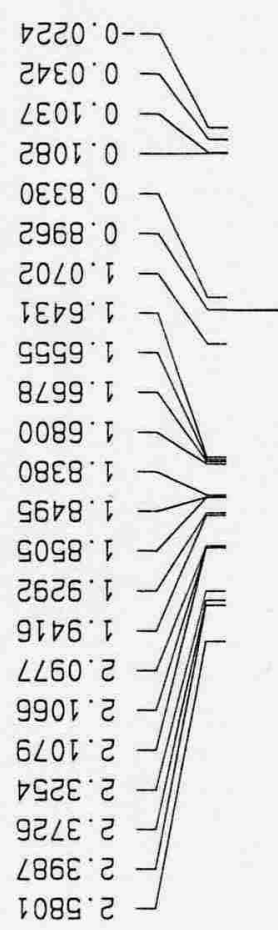

6E૬ $L^{\circ} \triangleright$

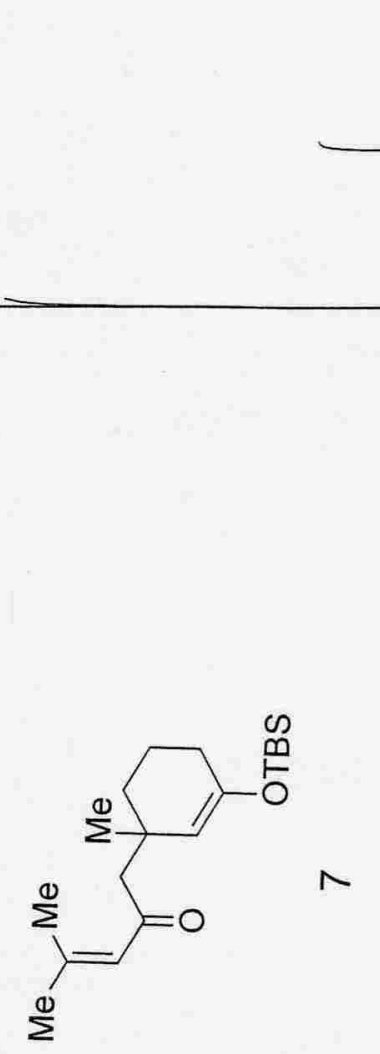

ह9॰0. 9

I9ट2' 9

(n)
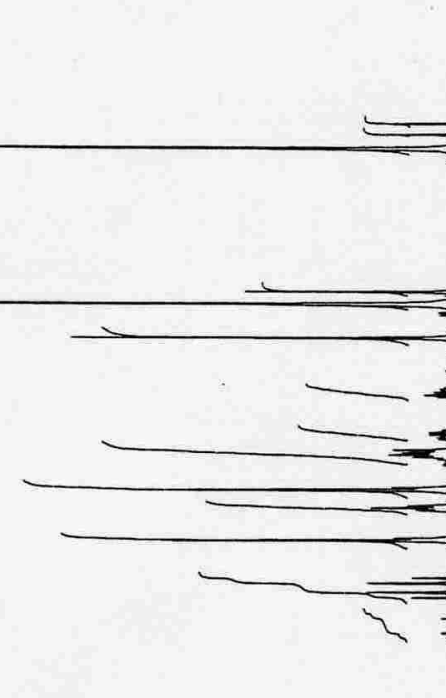

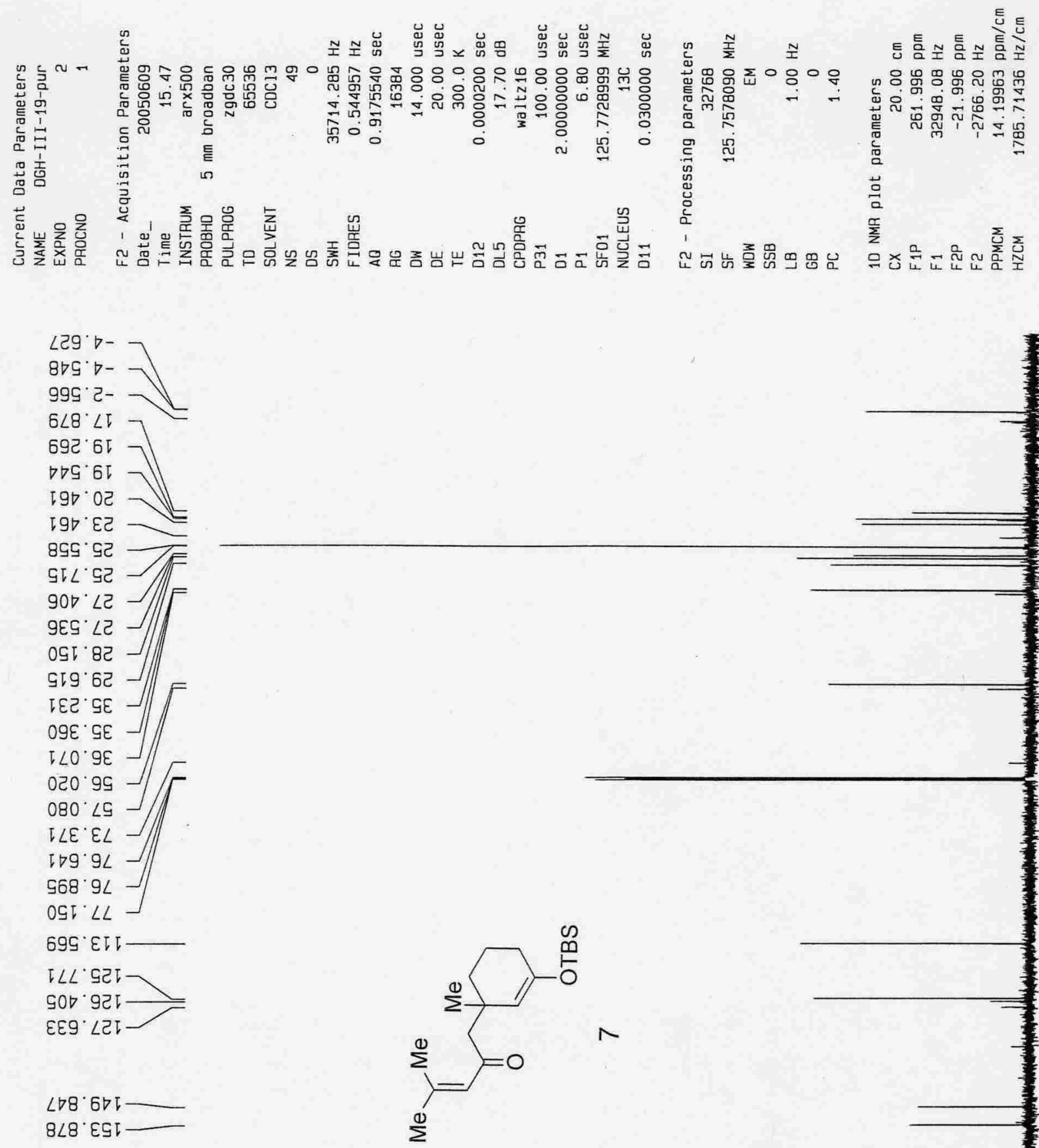

$665^{\circ} 002$ 

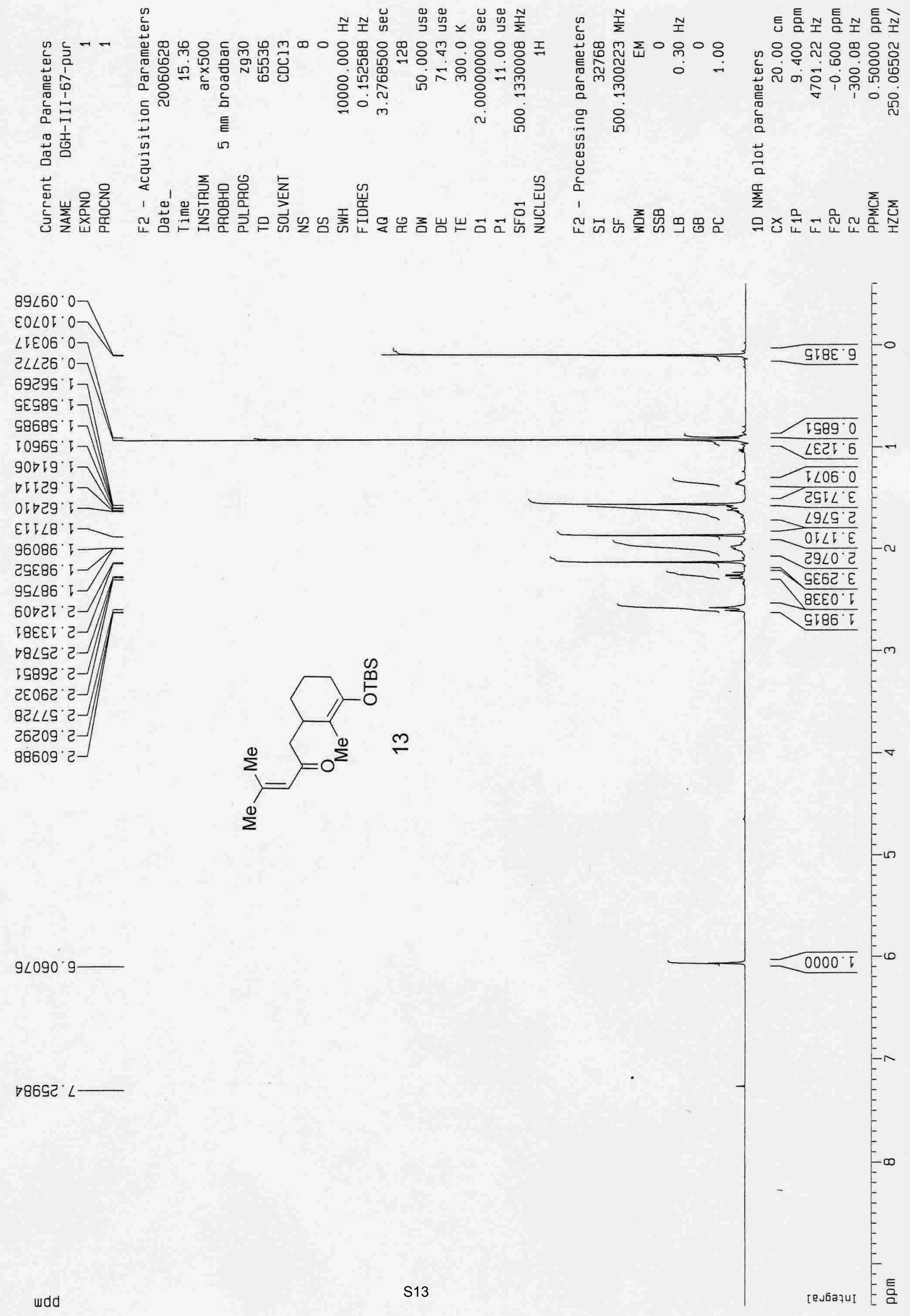

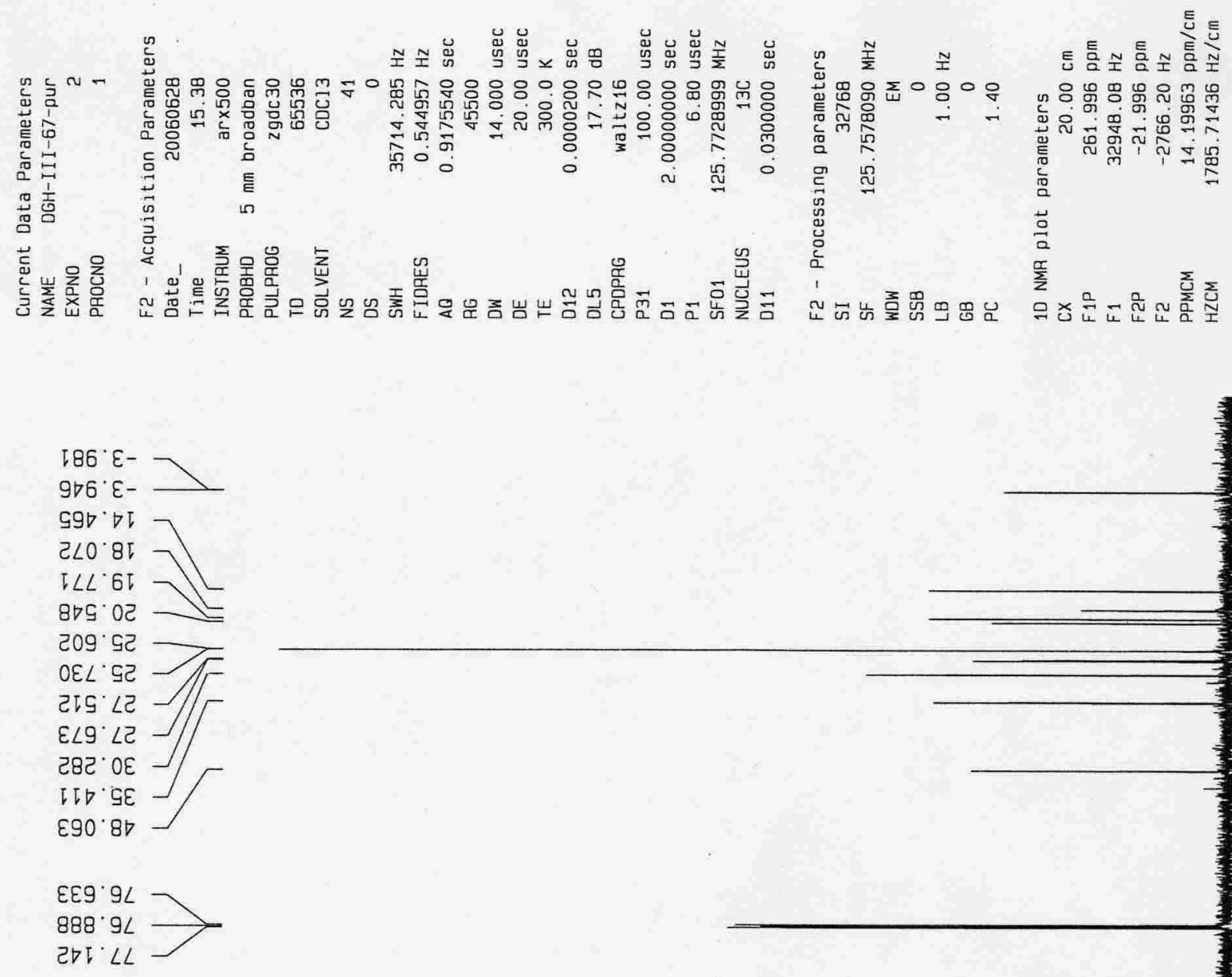

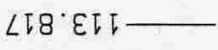

$600^{\circ}$ 口टा一

8૬จ $\nabla \nabla \Gamma$

$20 L^{\circ} \nabla 9 \Gamma$

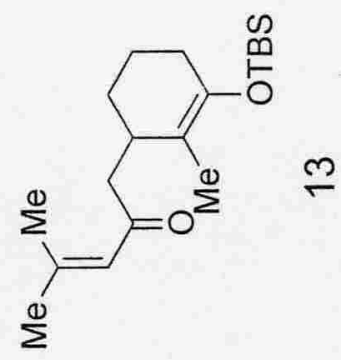

$\varepsilon \angle 6^{\circ} 002$

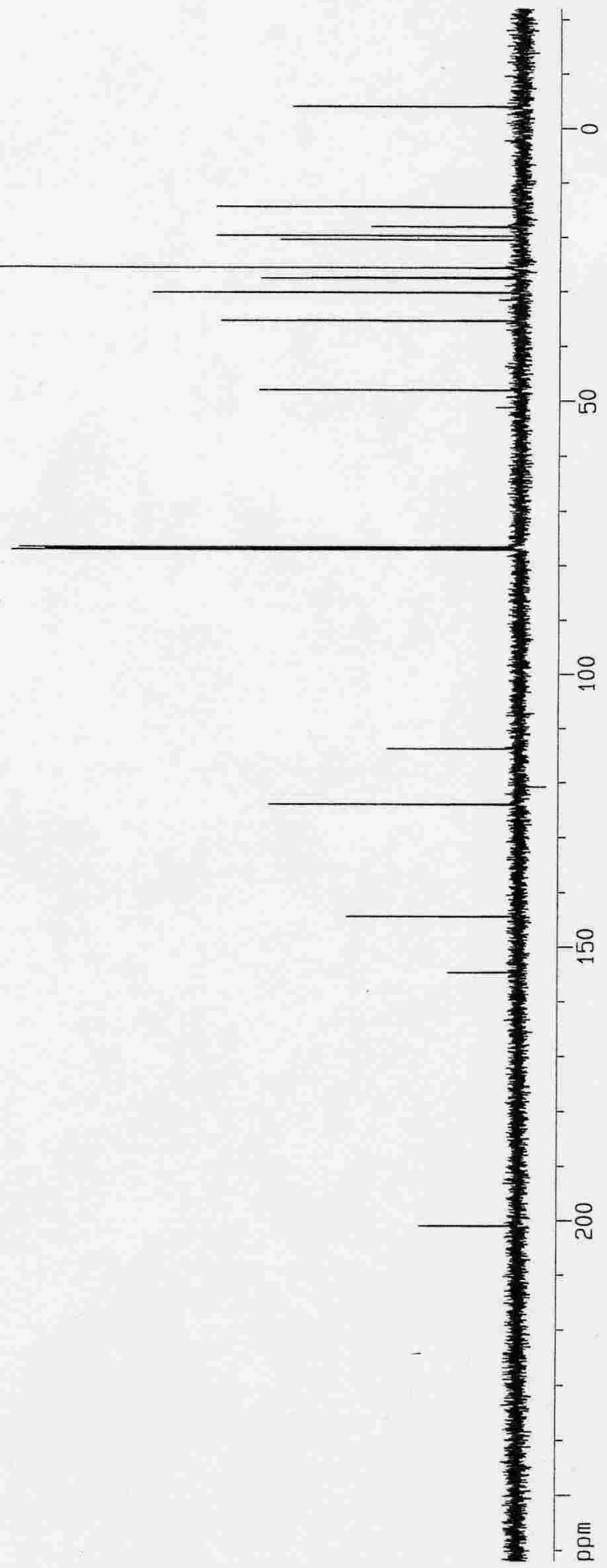



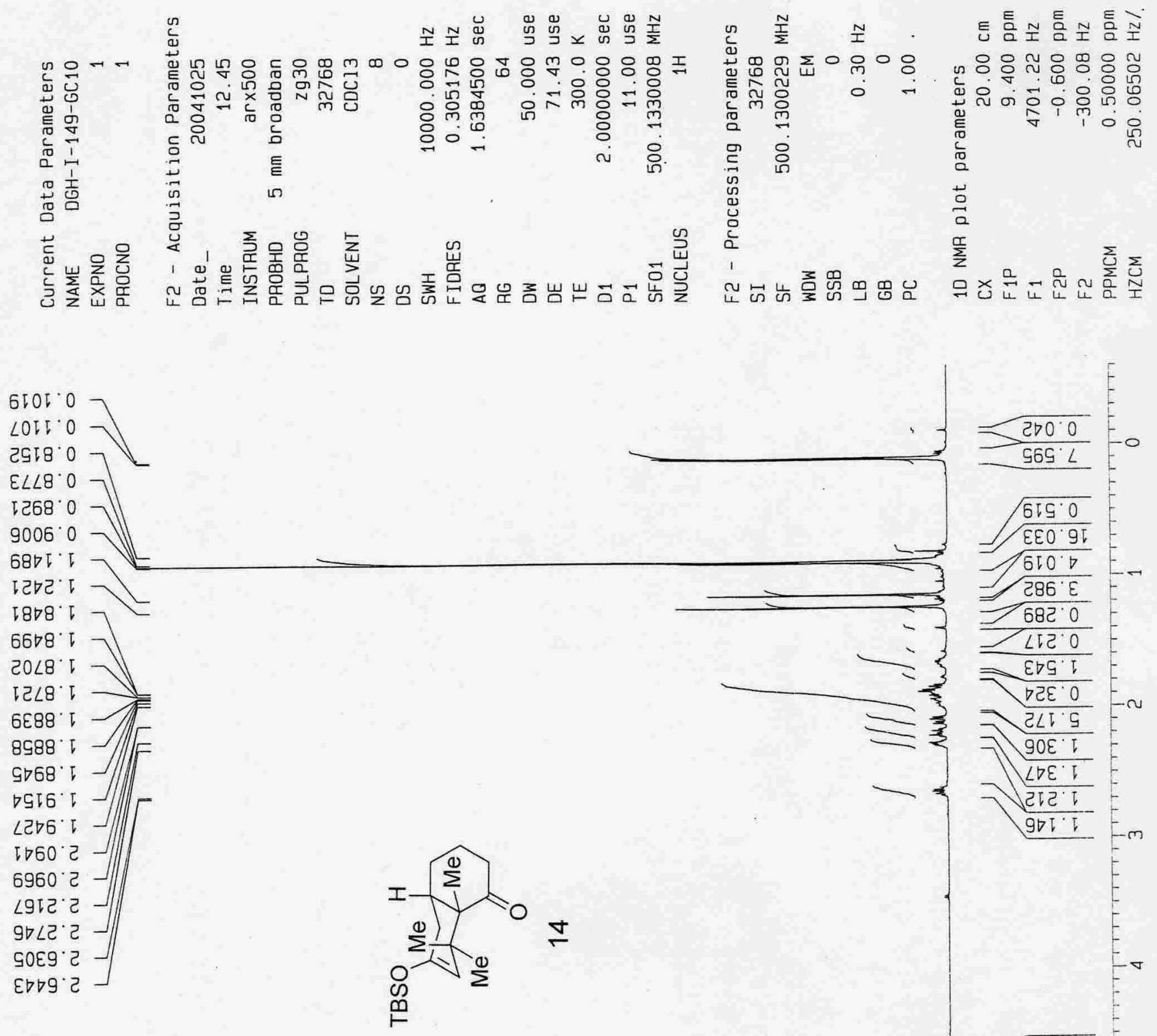

IIE9

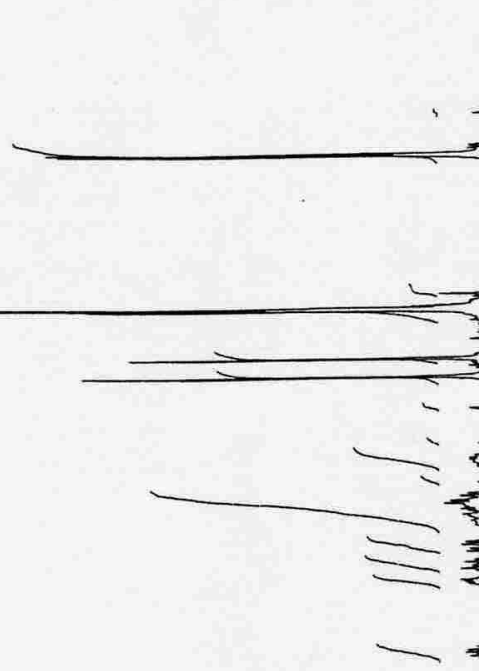



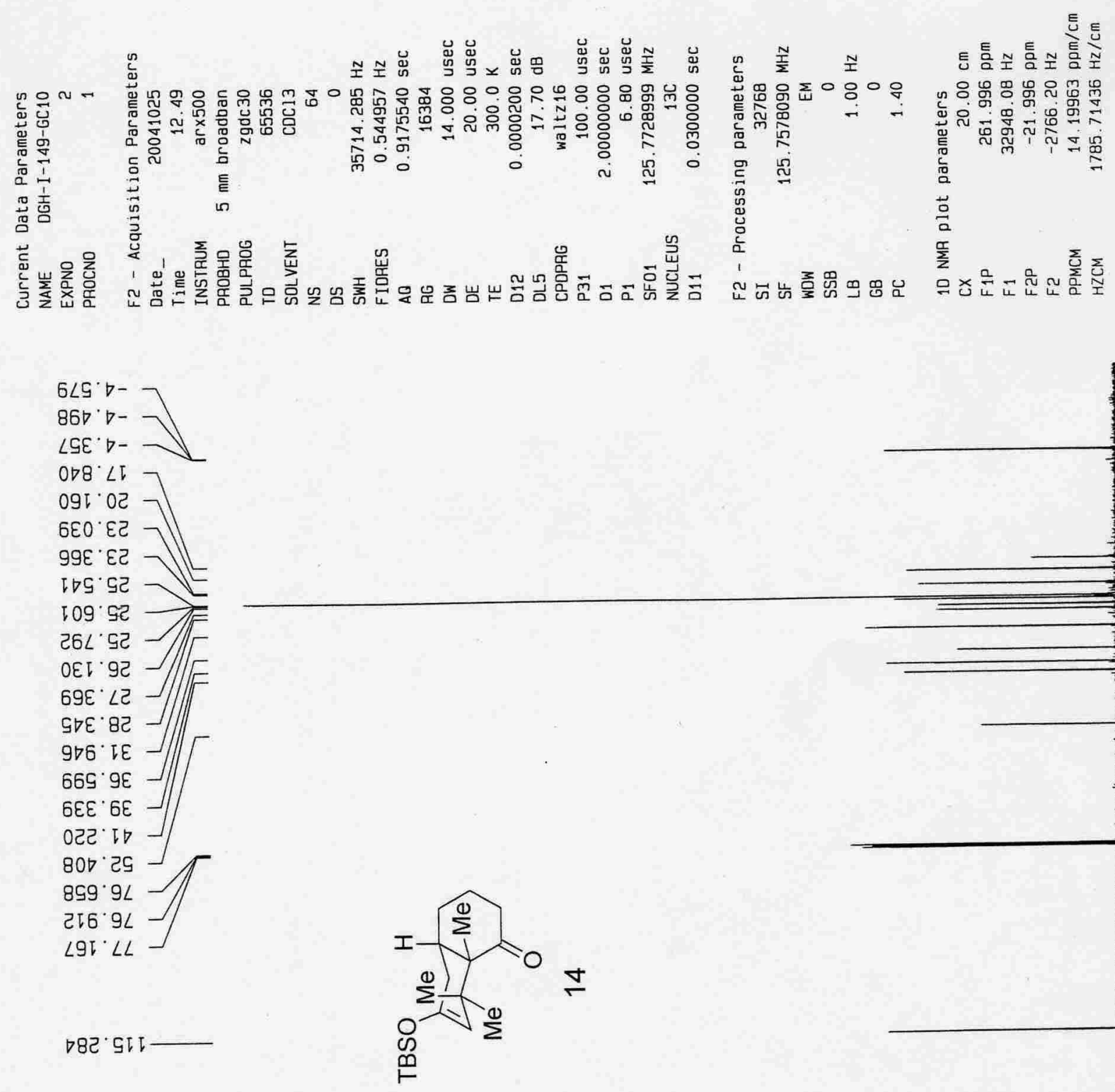

$\nabla \varepsilon 6 \cdot \nabla \nabla \downarrow$

8८० $\varepsilon I ट$ 


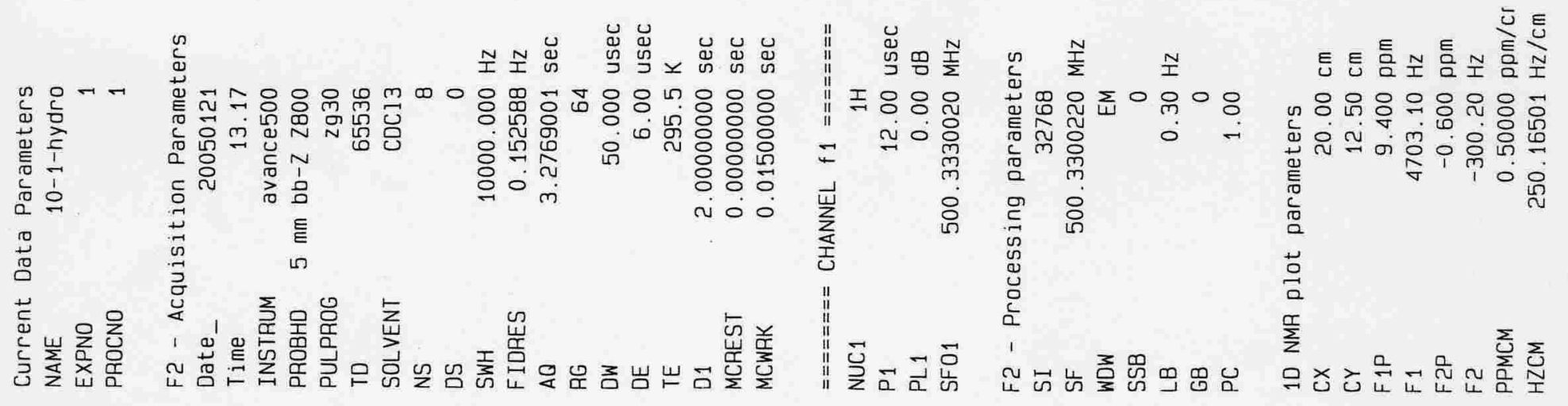
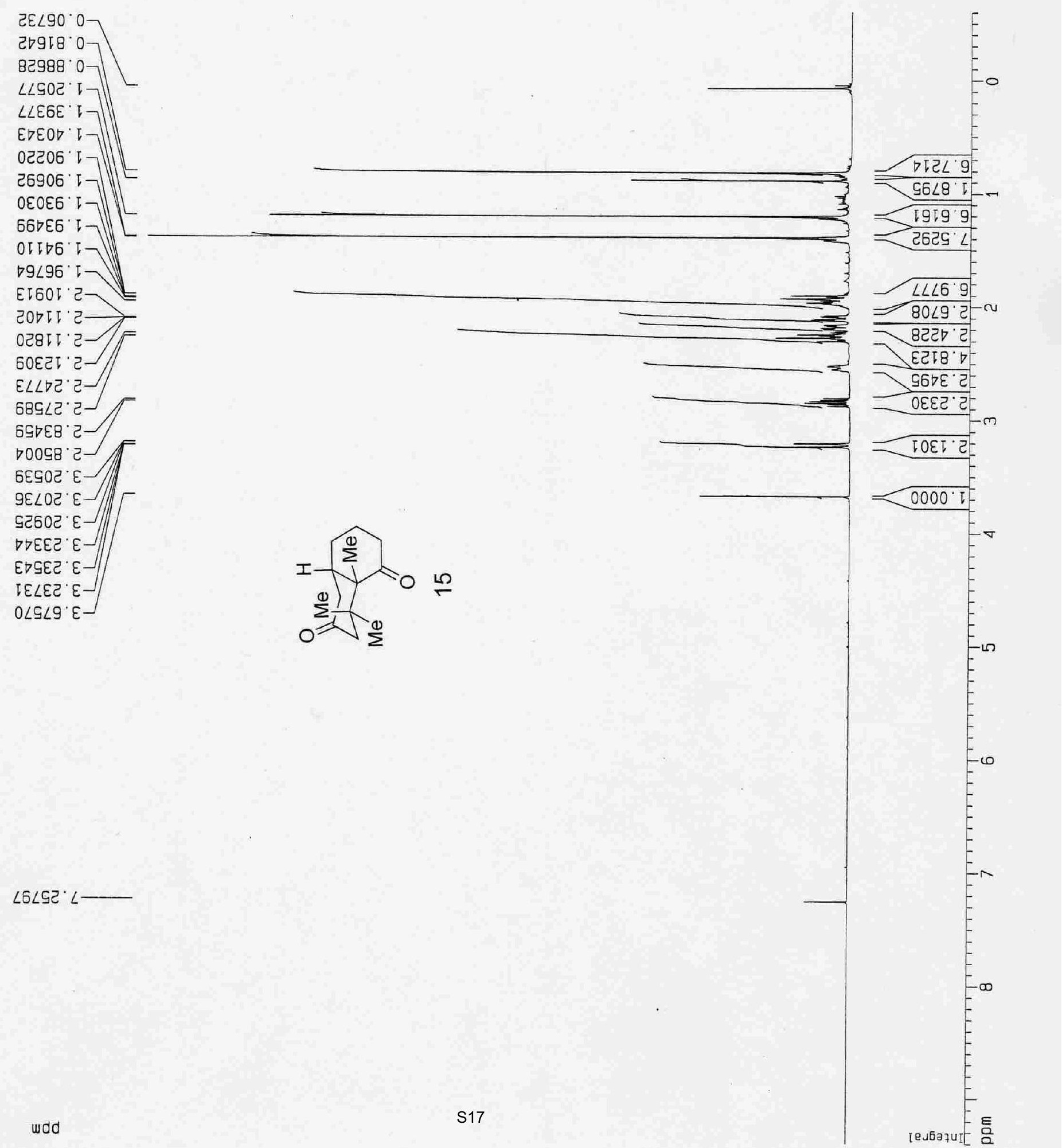

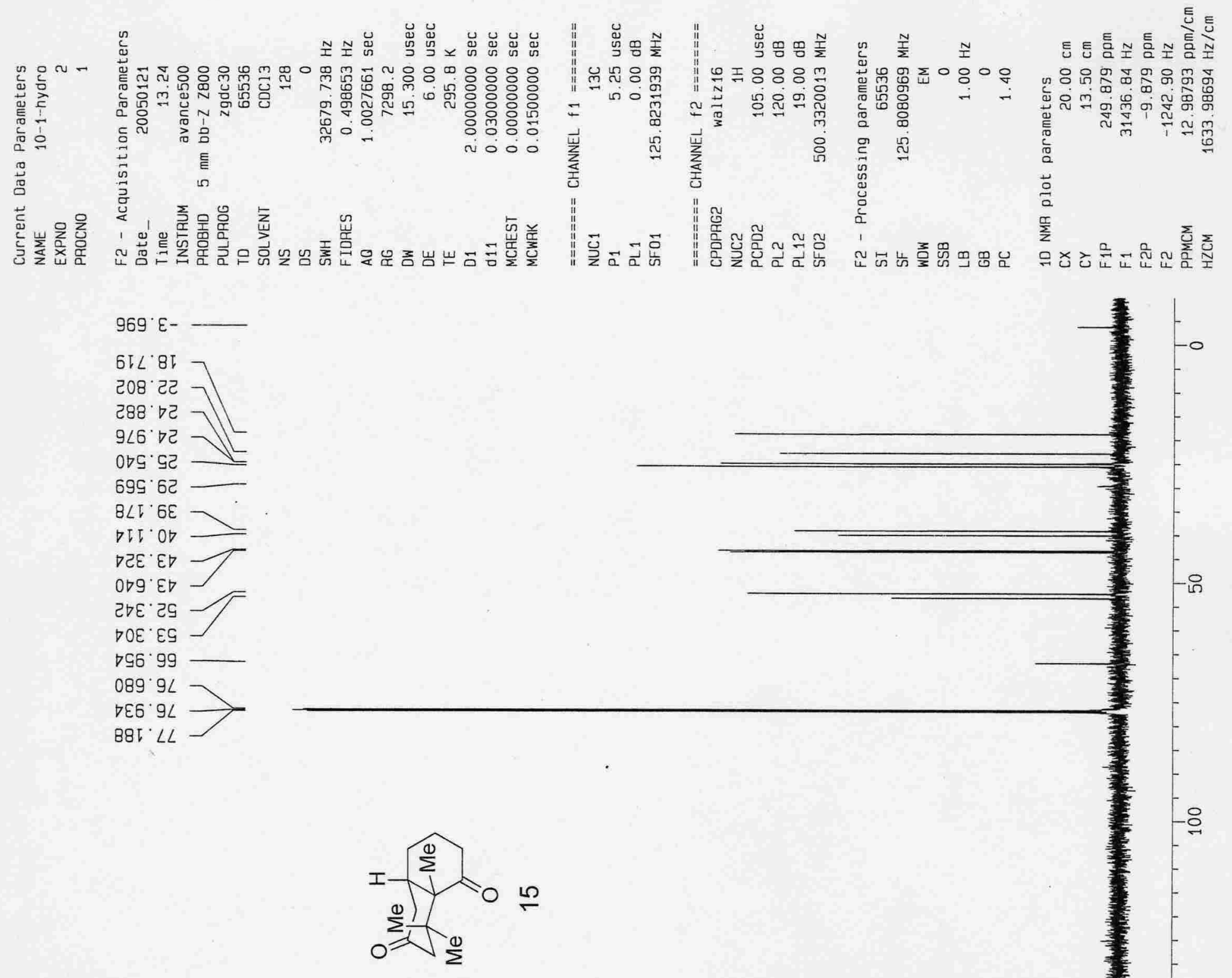

OEट Itน-

$8 \varepsilon 9 \cdot \nabla \amalg 己-$ 

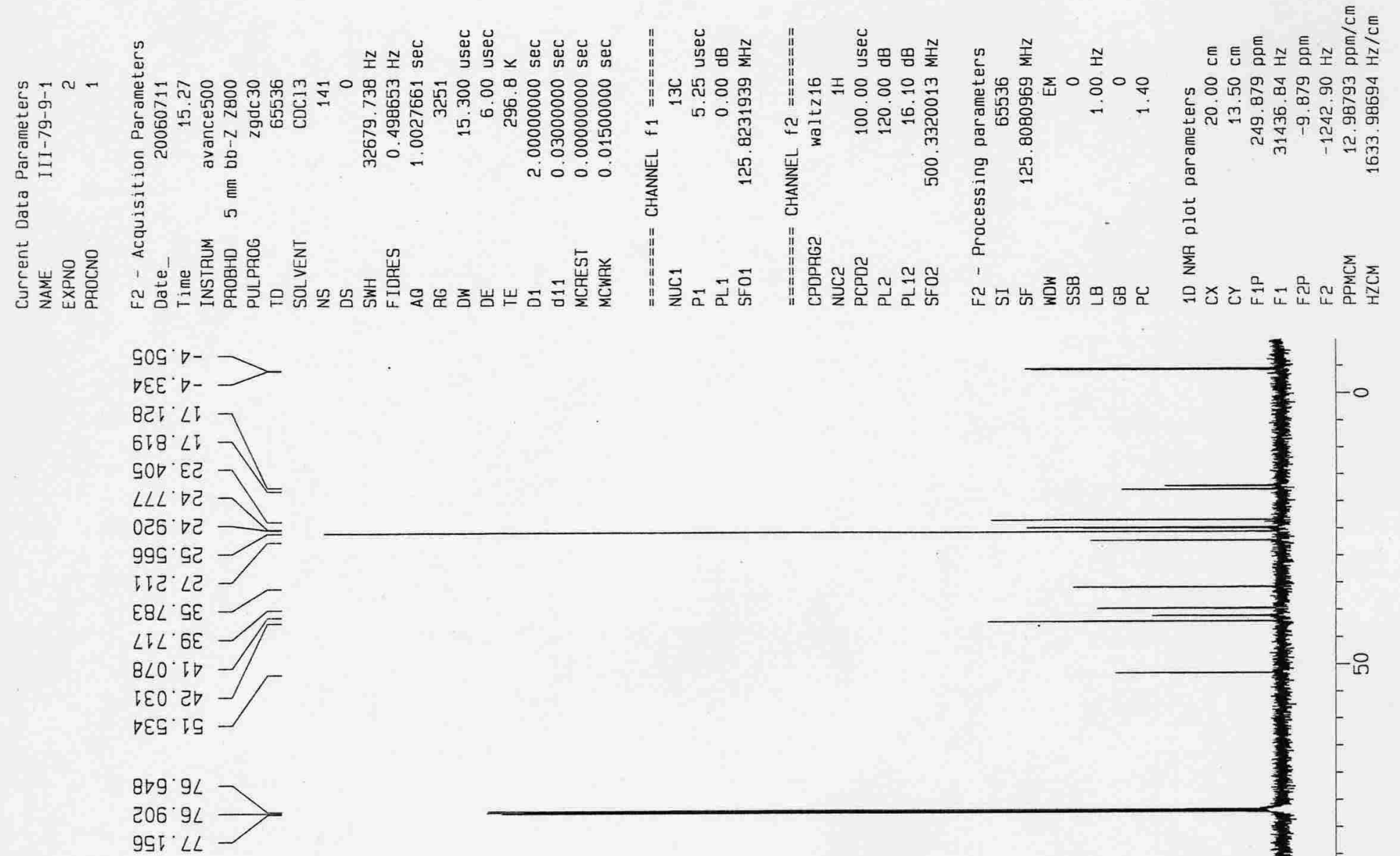

$\angle \nabla D^{\circ} \angle O T-$

Е૪0. ट૬โ

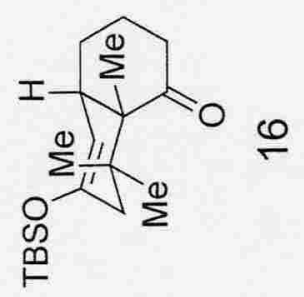



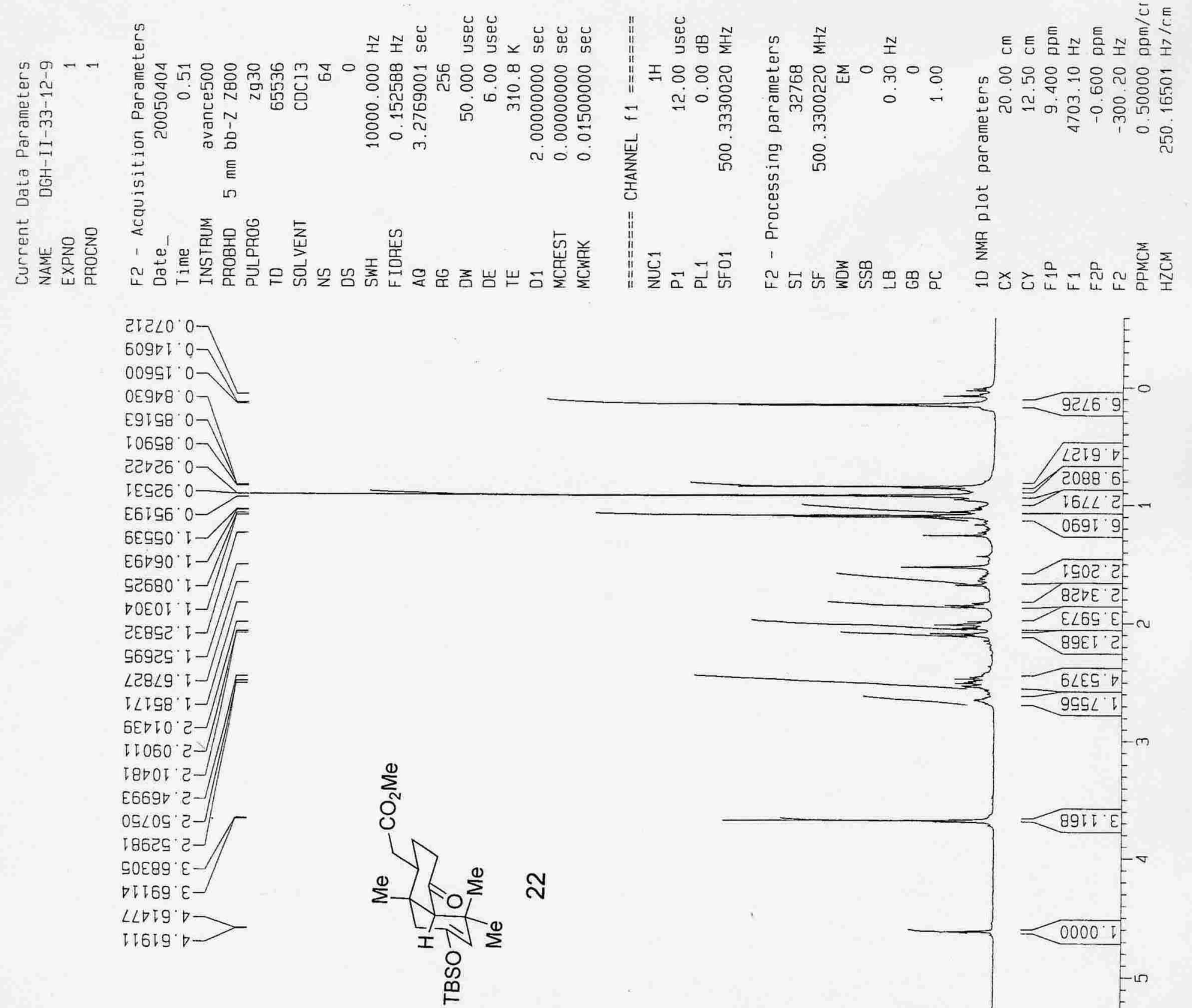

6r092 $\angle \longrightarrow$

9900E $\angle \square$

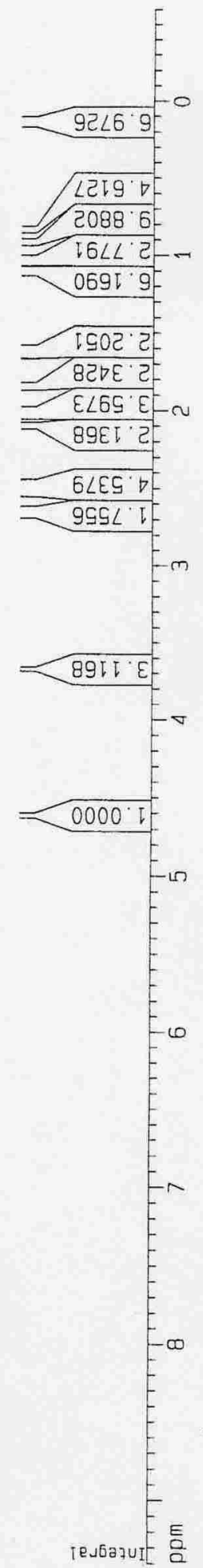



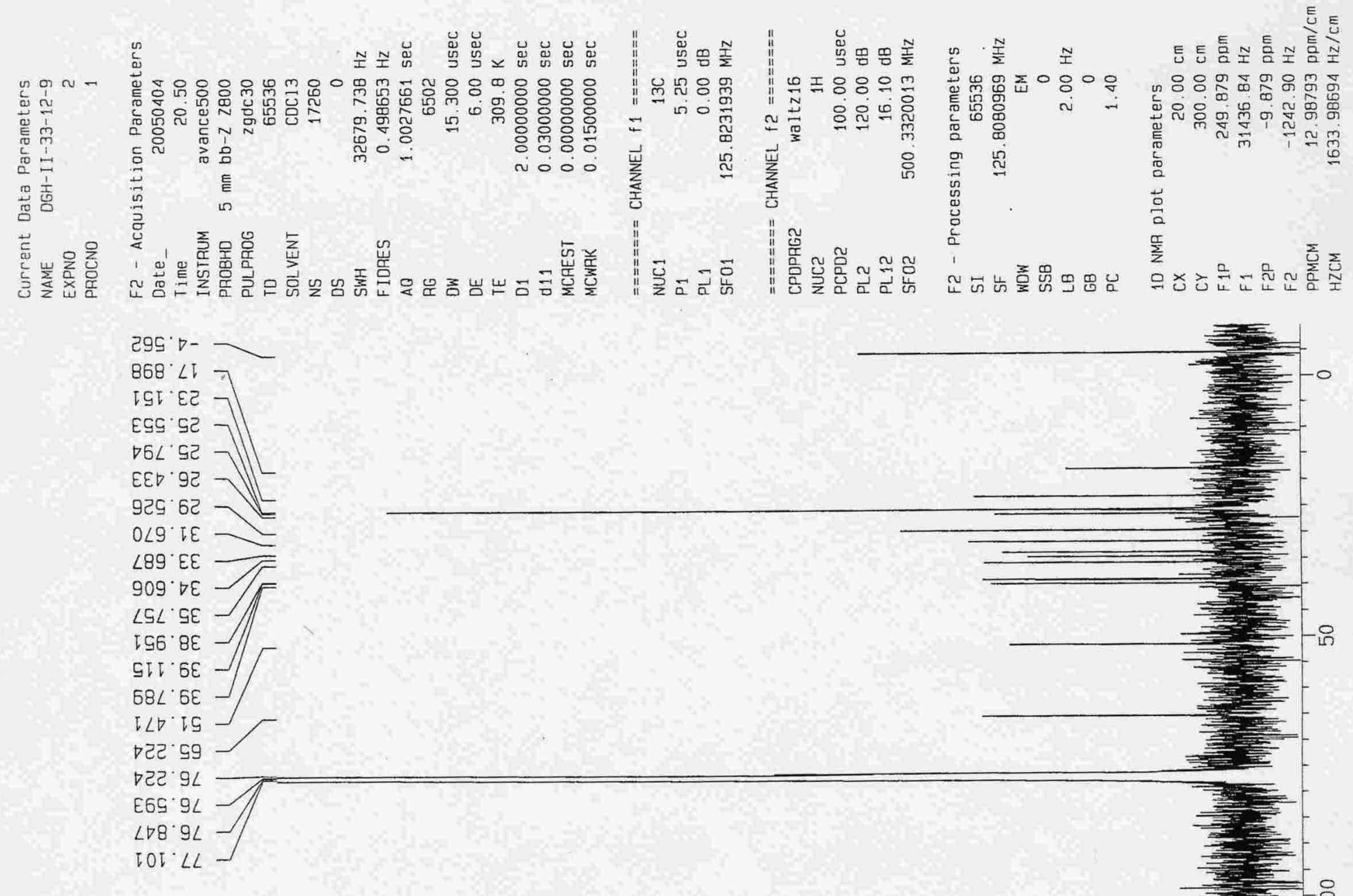

LECEIT-

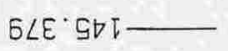

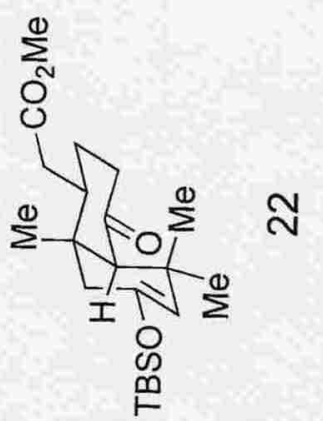

$\angle O Z^{\cdot} \varepsilon \angle T-$

$\varepsilon \nabla \downarrow \varepsilon I 己-$ 


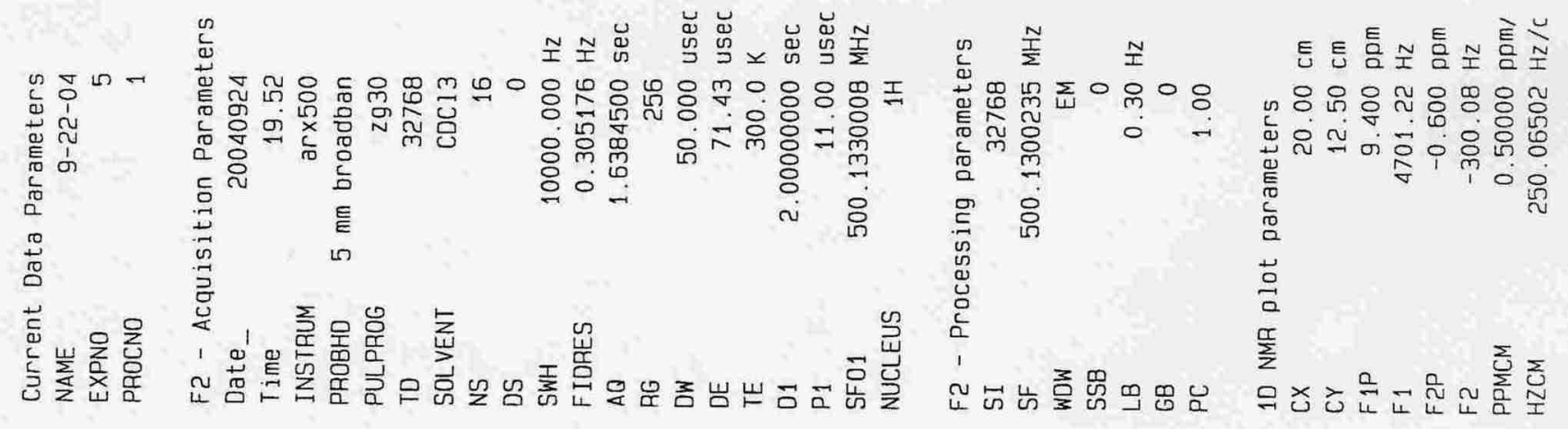
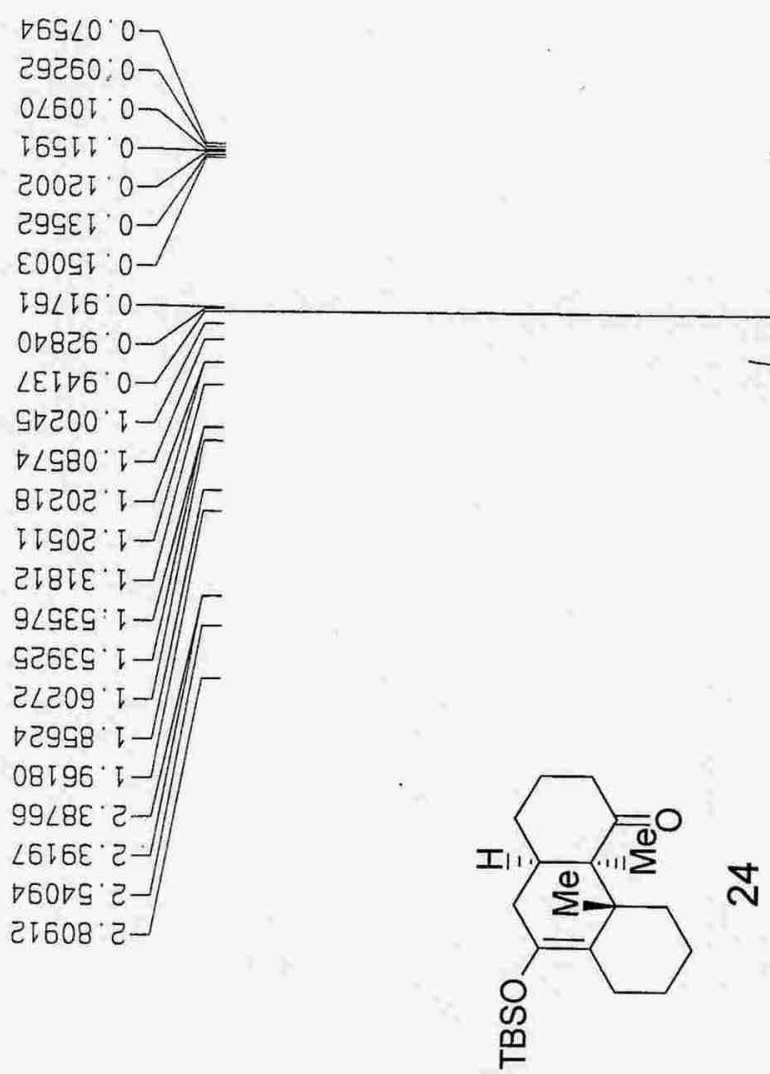

$60092^{\prime} \angle \square$ 


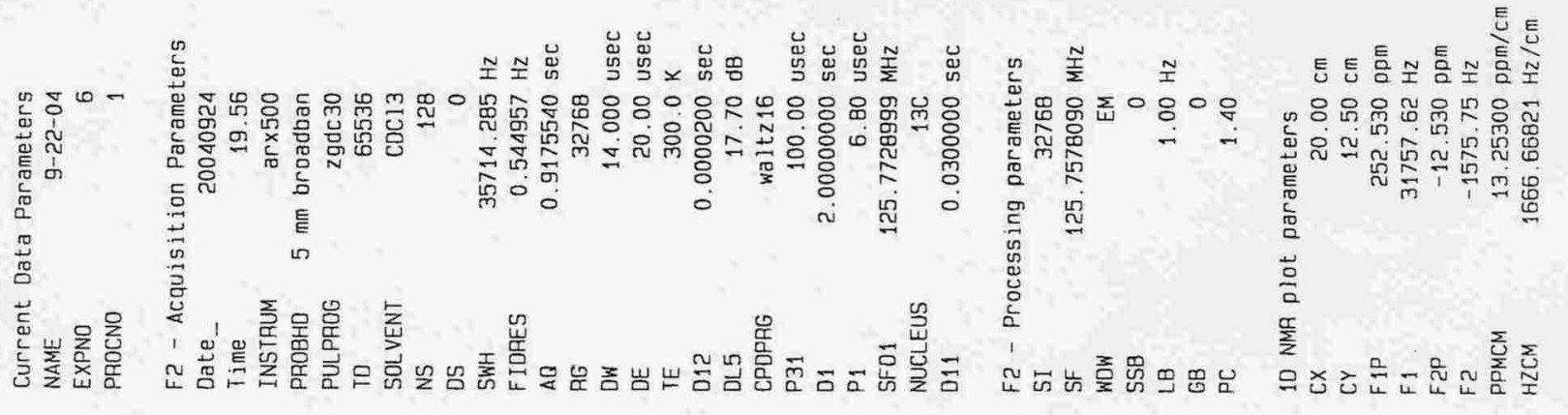

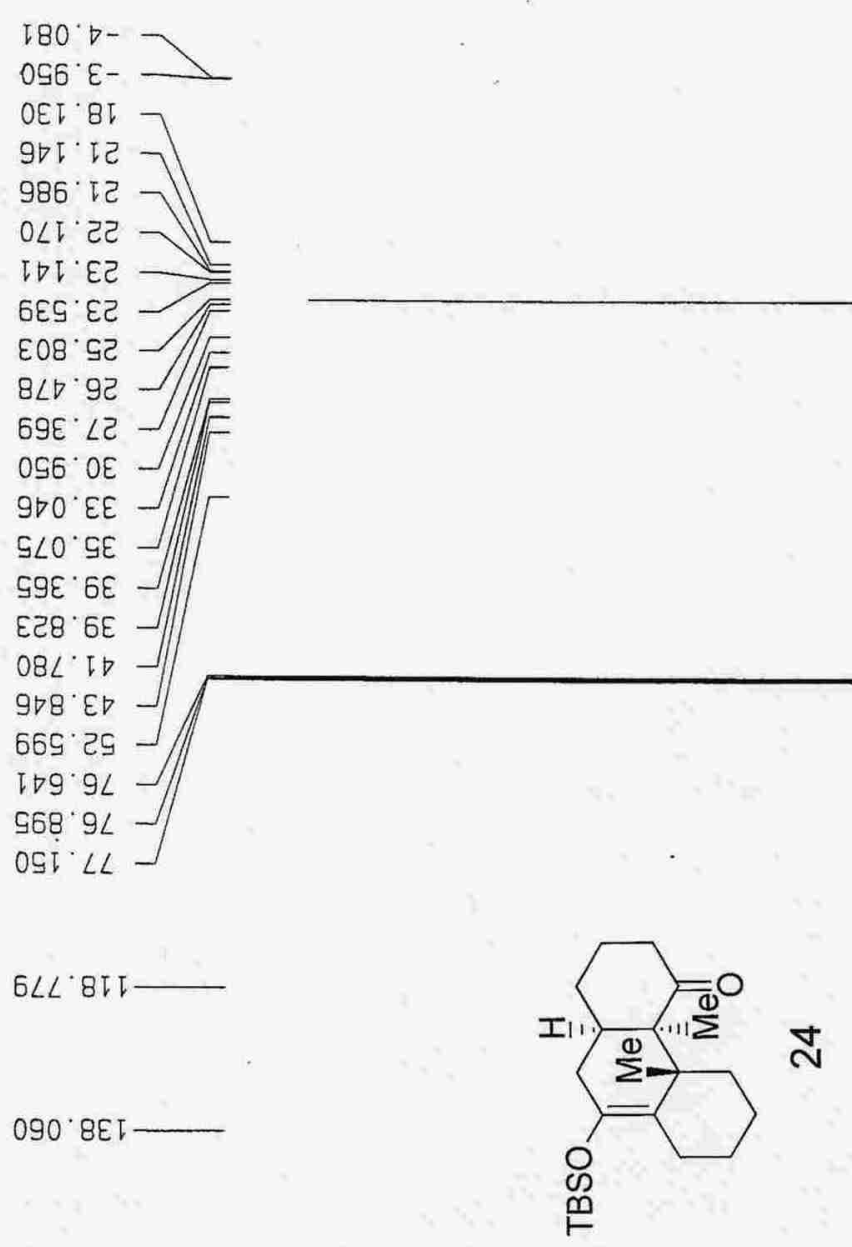

99G SIL 

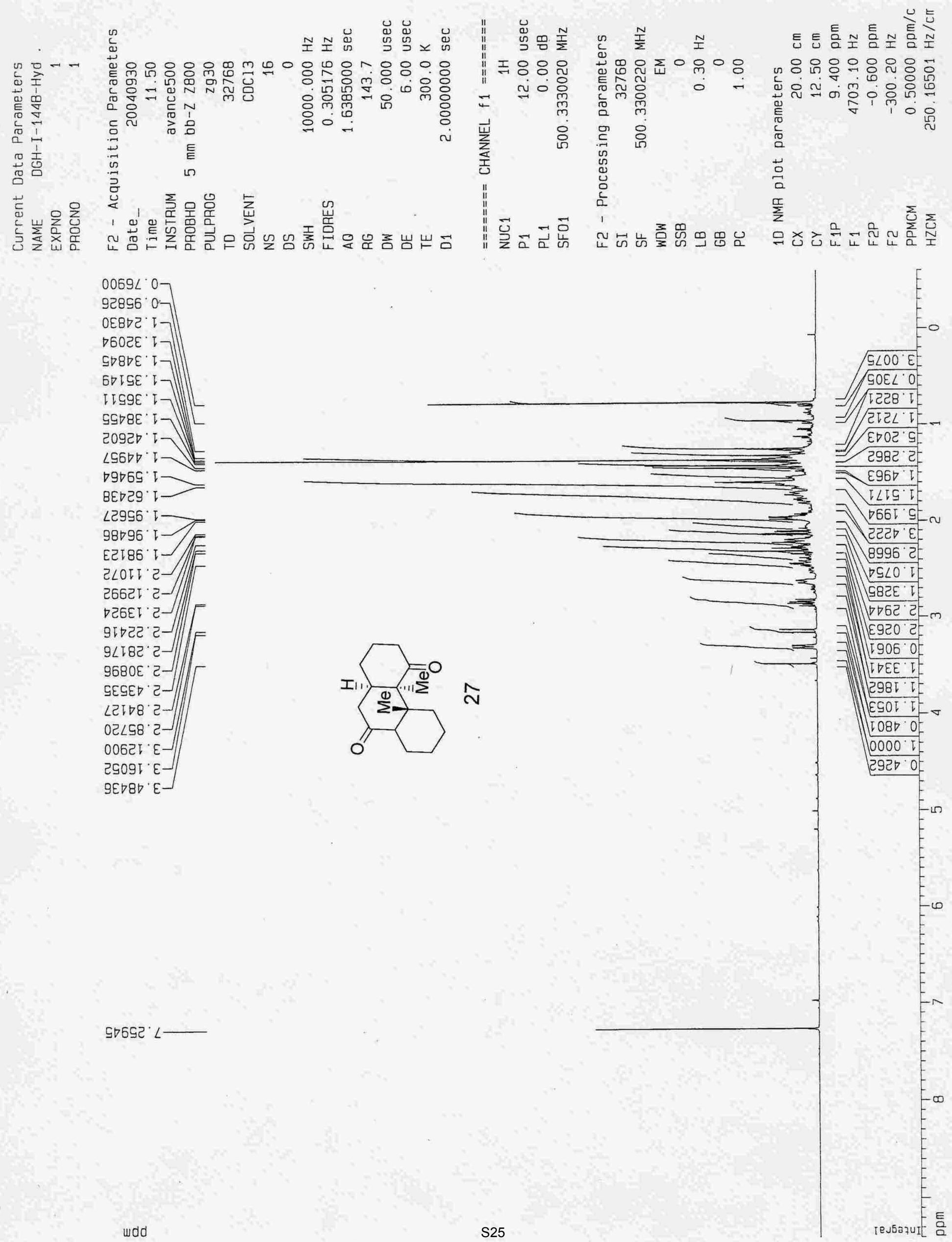


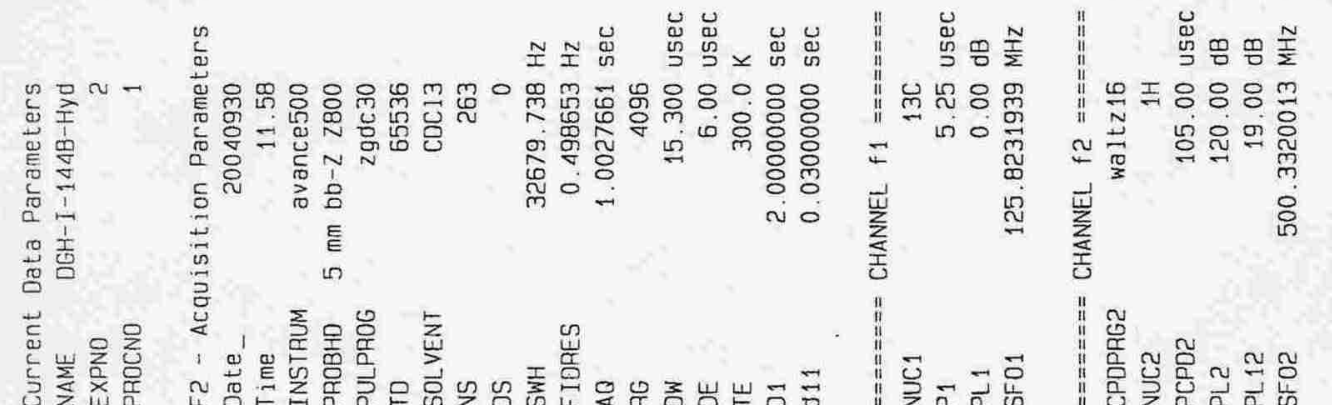

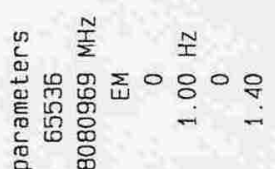

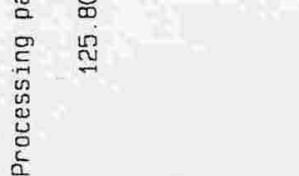

产

튼

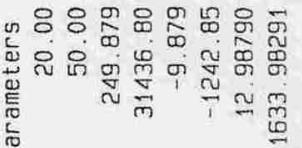

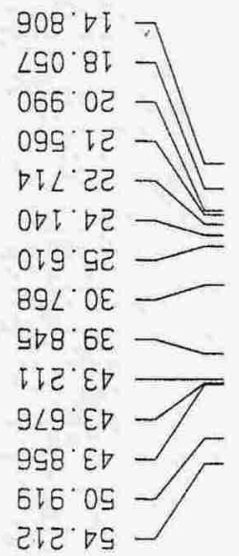

$\nabla \nabla 9^{\circ} 9 L$

$868^{\circ} \cdot 9 L$

टII $L L$

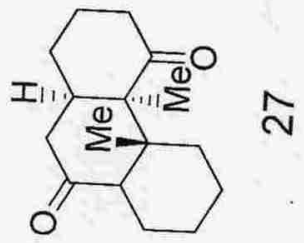



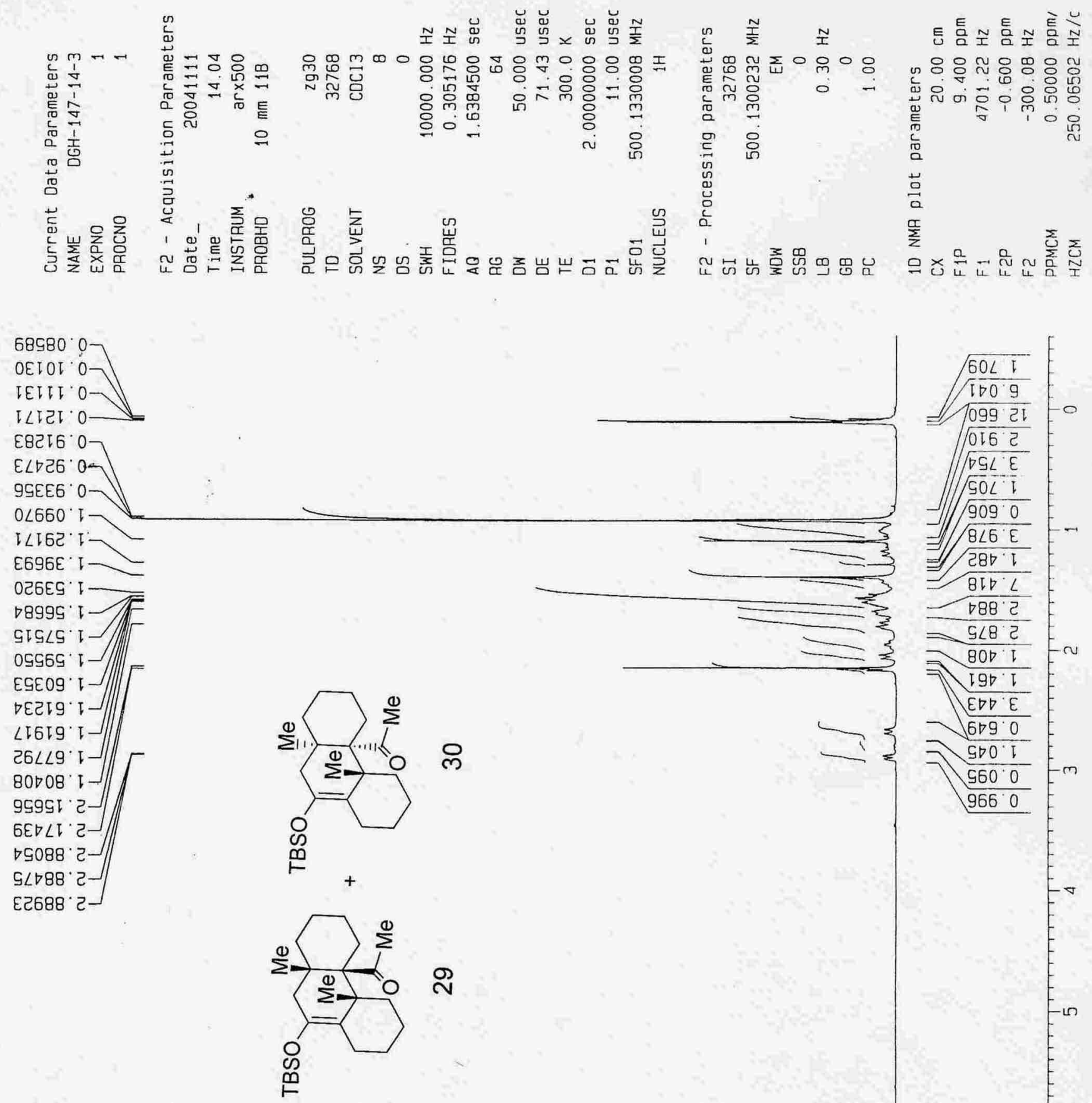

$60092^{\circ} L$
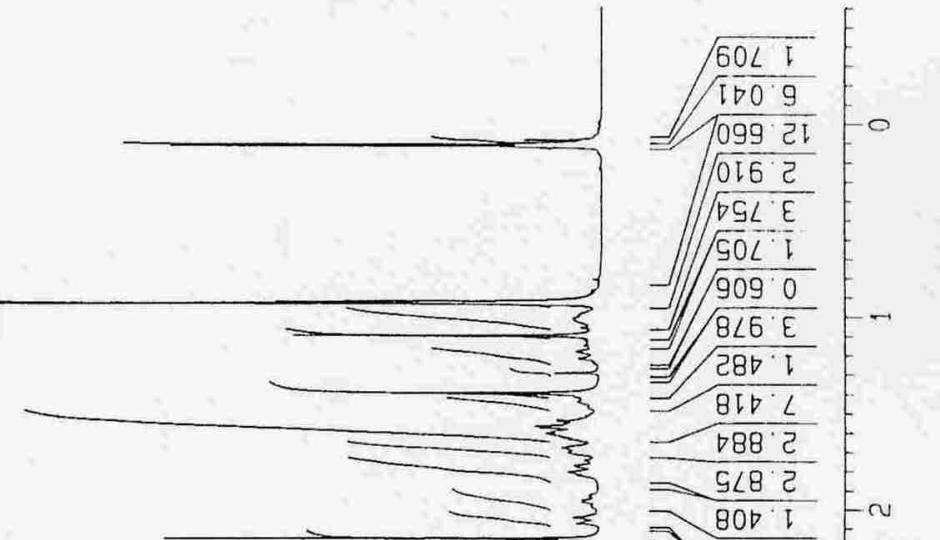

$\frac{909 \cdot 0}{8 \angle 6 \cdot E}=-$
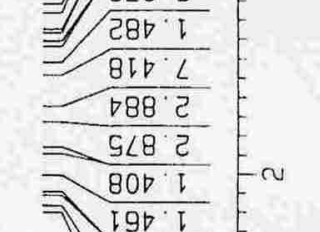

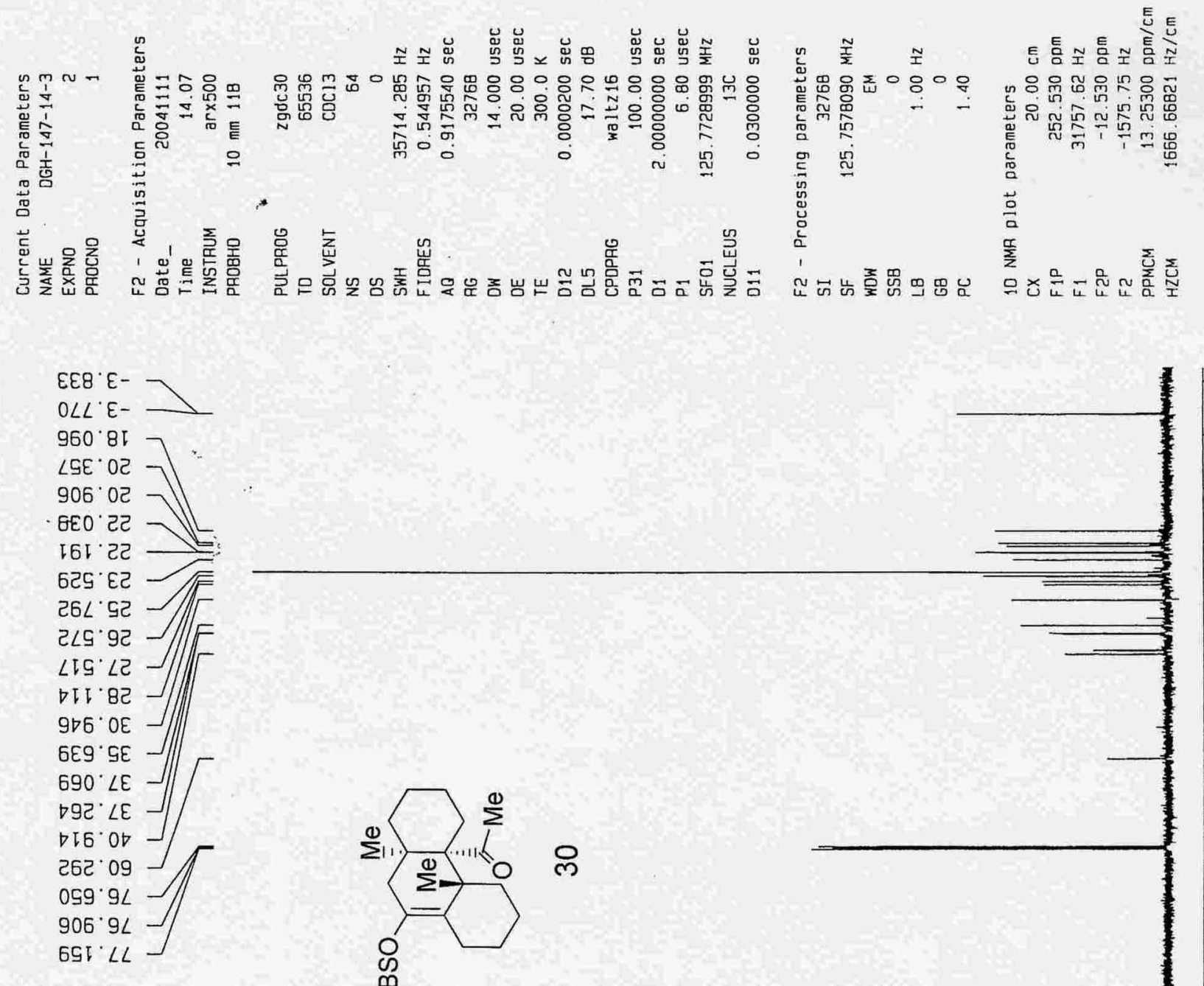

टЕナ・8น

E60.6Еโ-

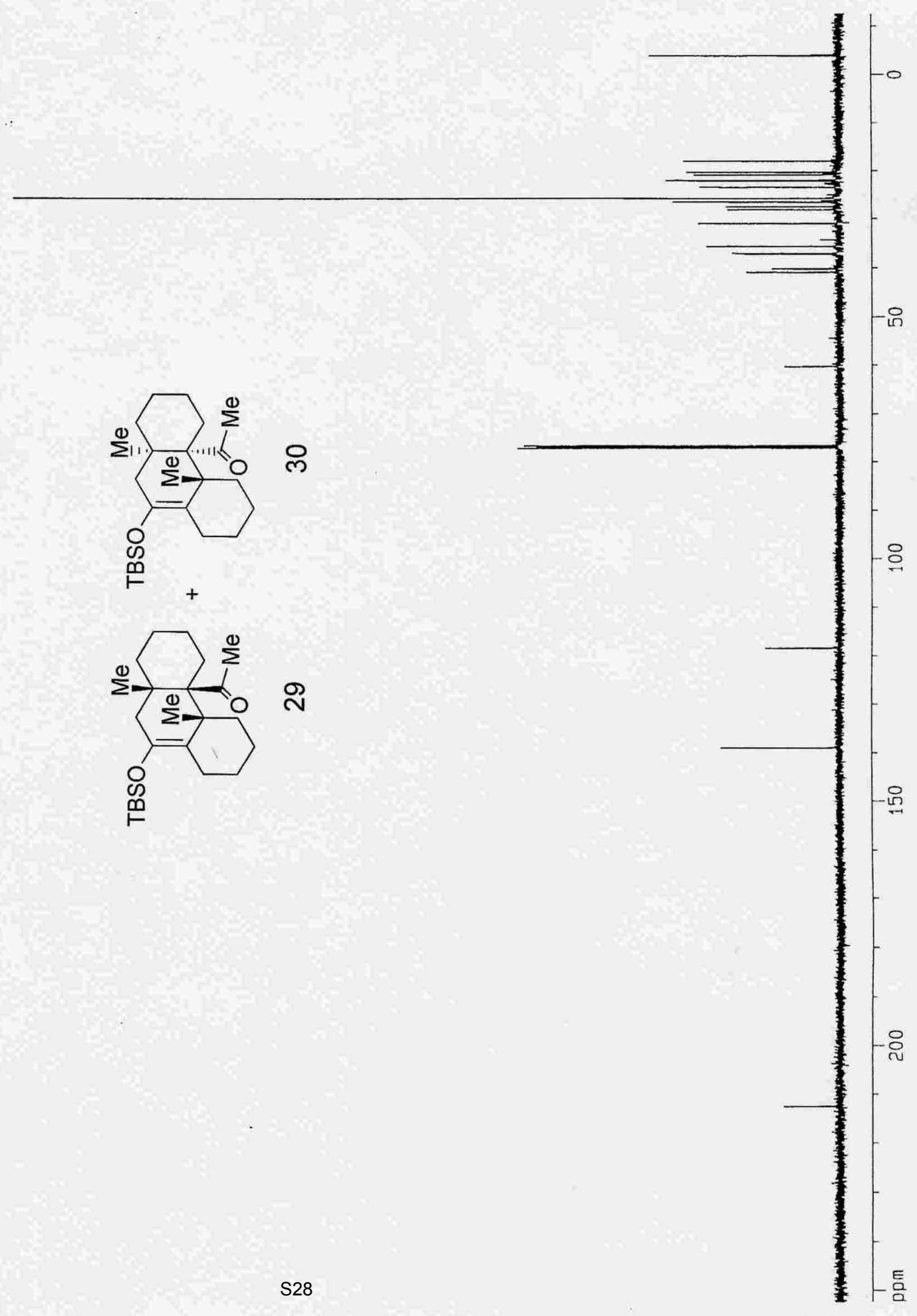



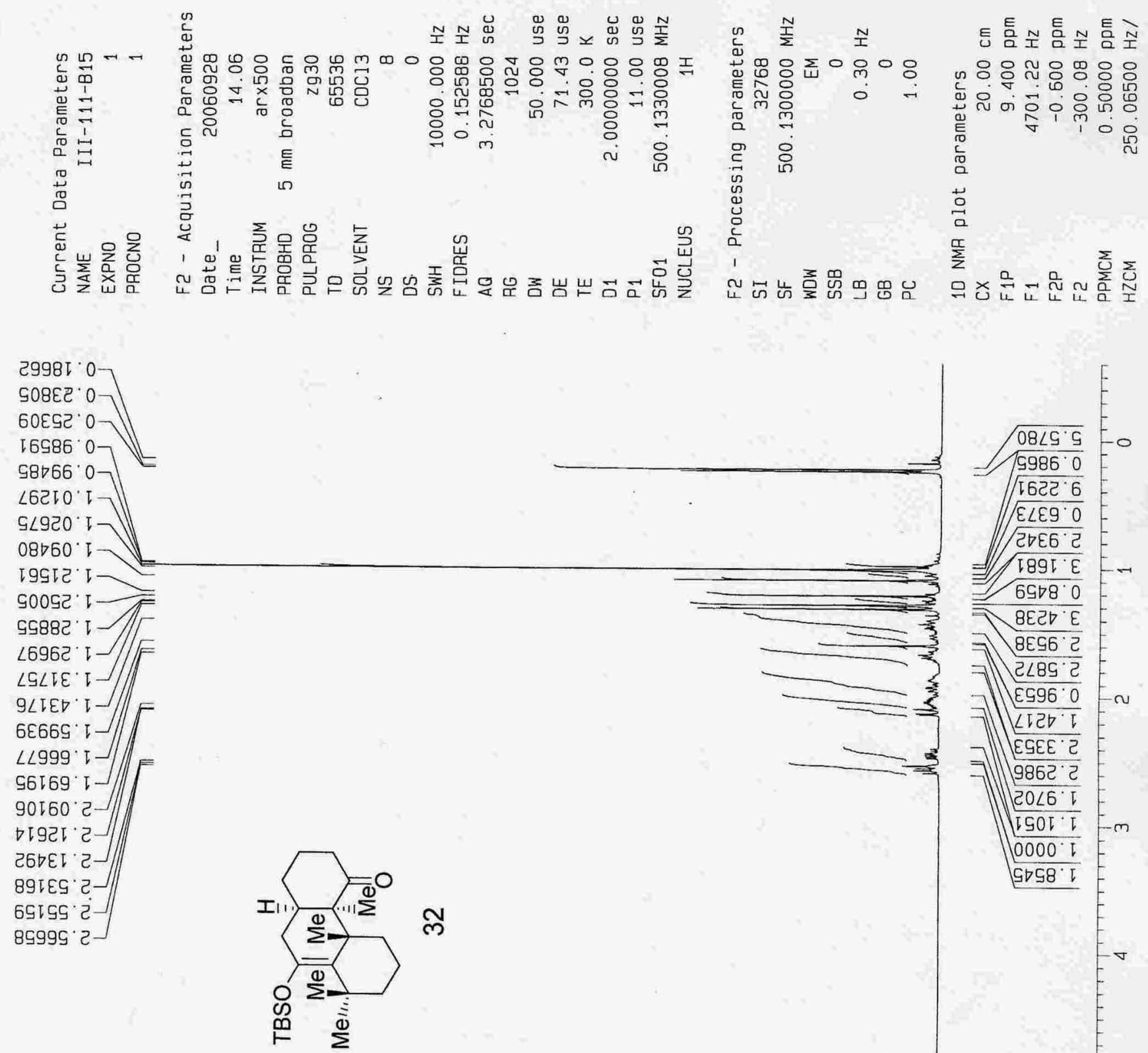

G9G0E $L$

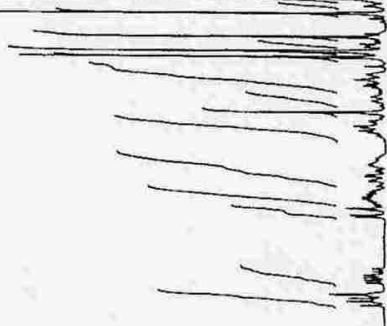



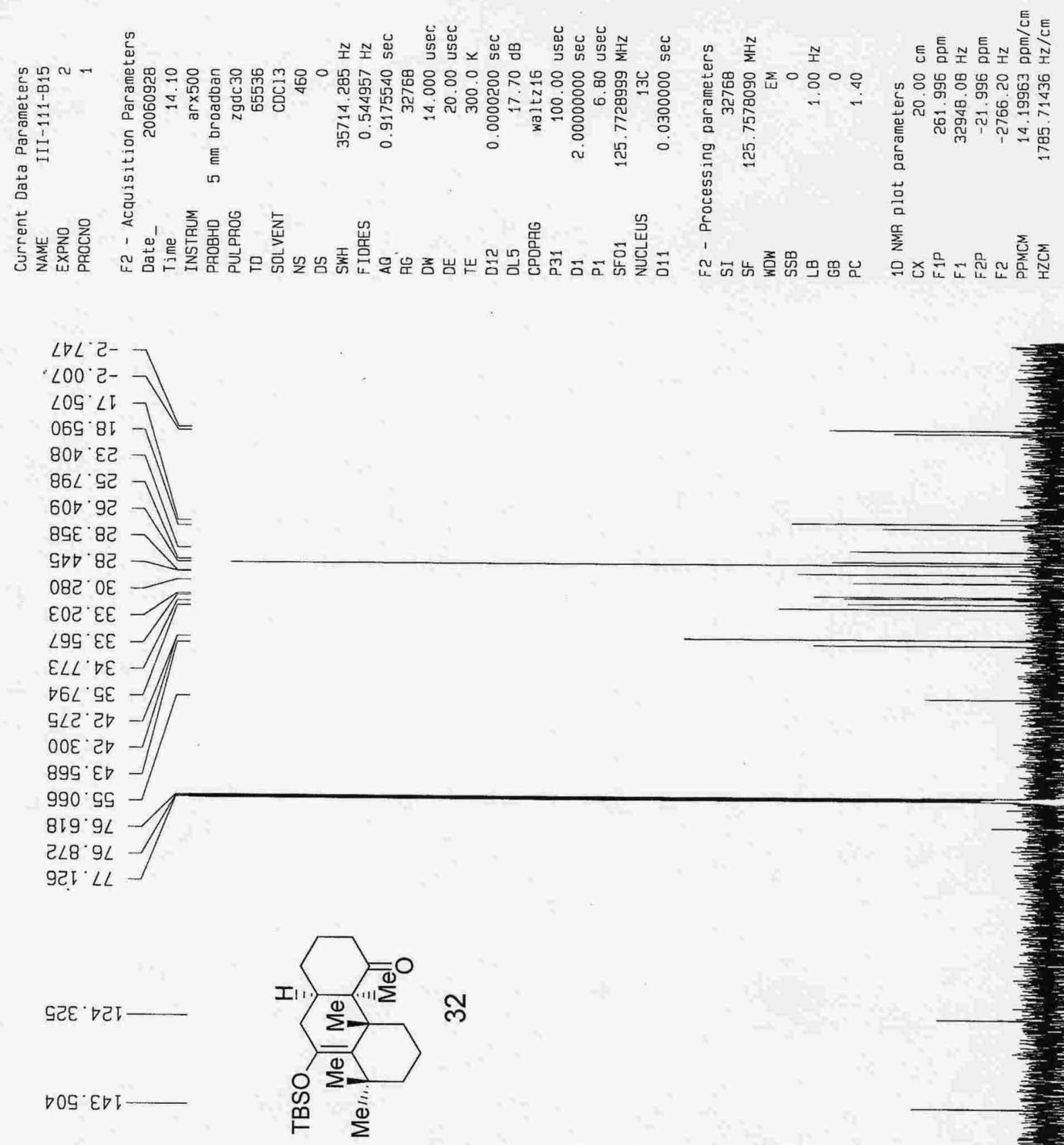


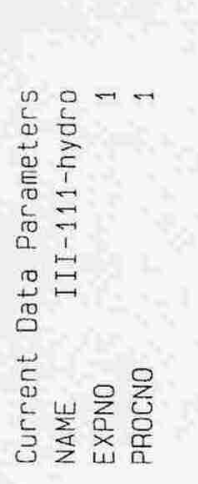

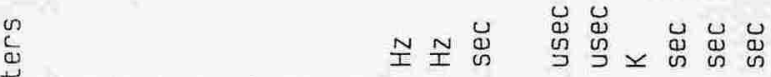

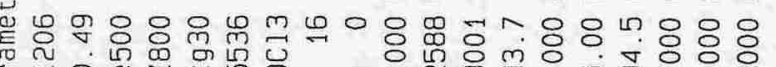

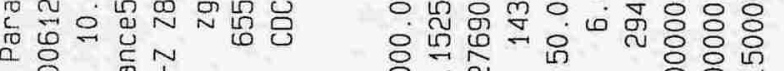

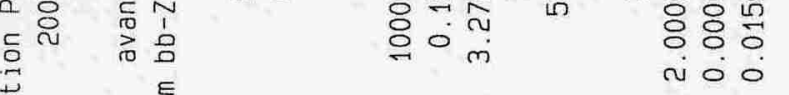

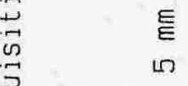

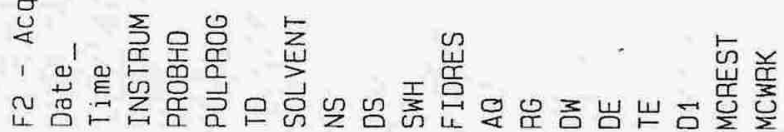

II

巡 $⿴ 囗 \frac{N}{2}$ 도웅요

ํํ유

iI

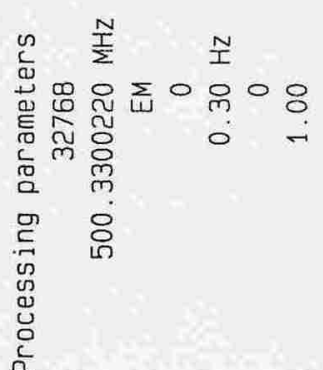

a

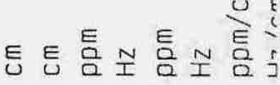

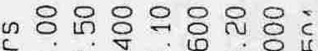

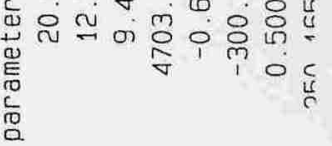

$\stackrel{\overrightarrow{0}}{a}$

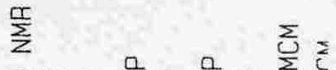

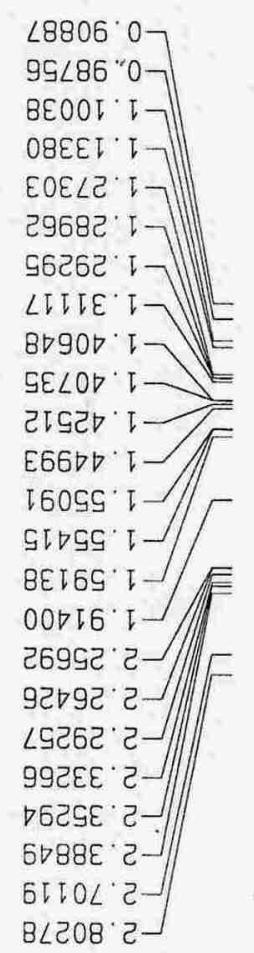

I $\angle 092^{\circ} \angle$
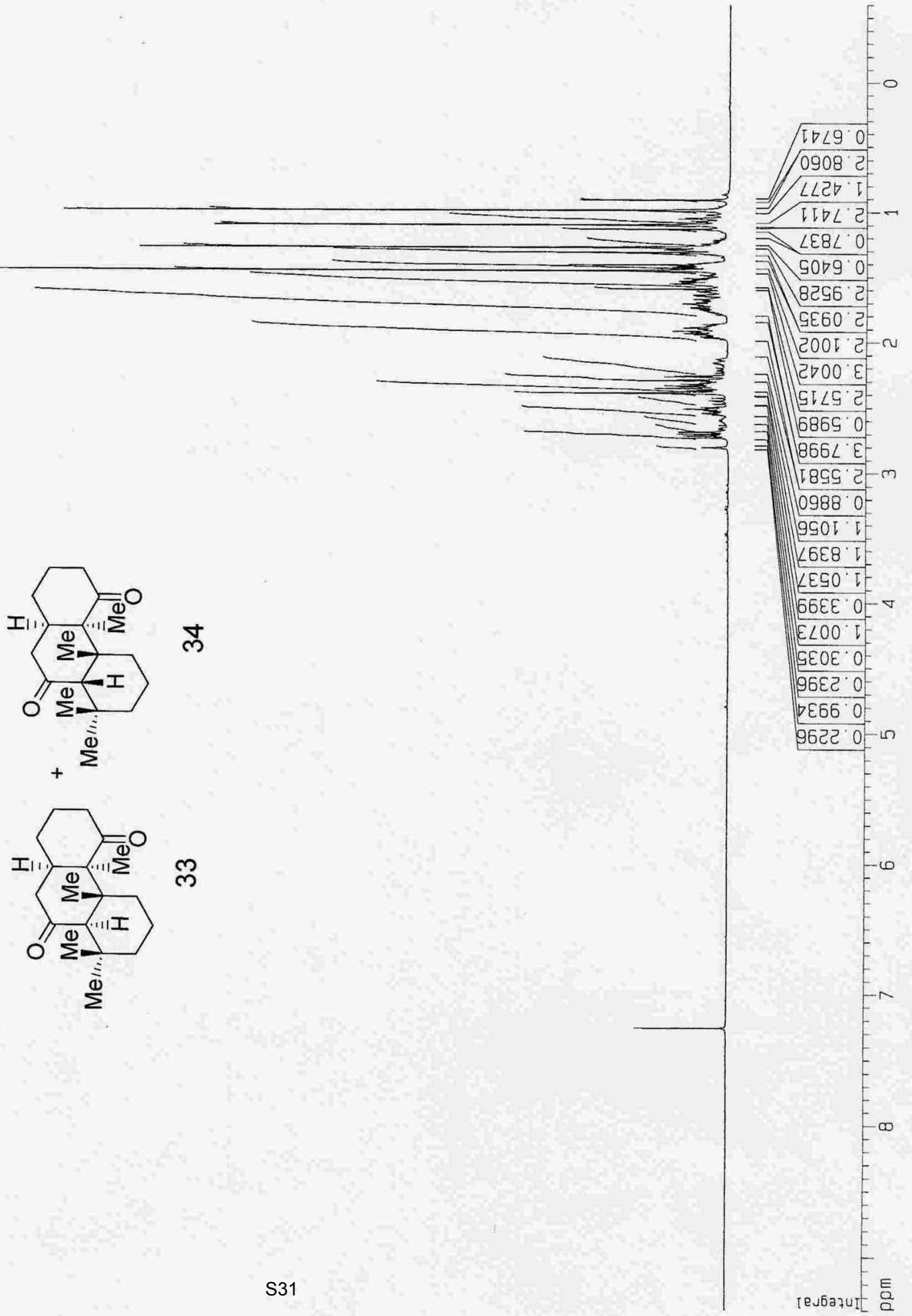

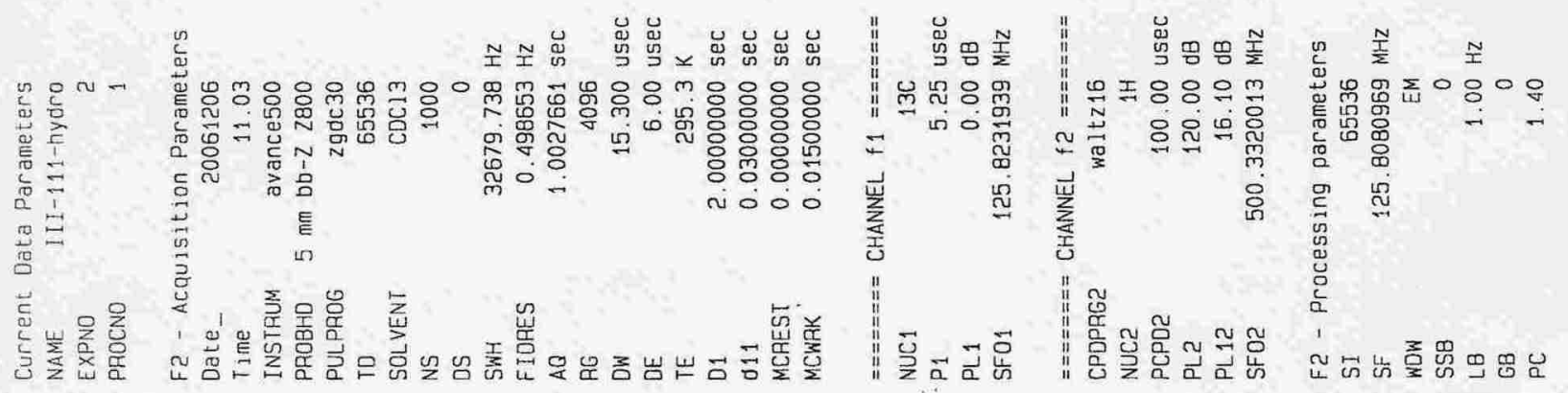

튼

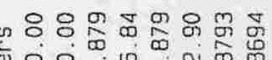

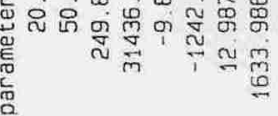

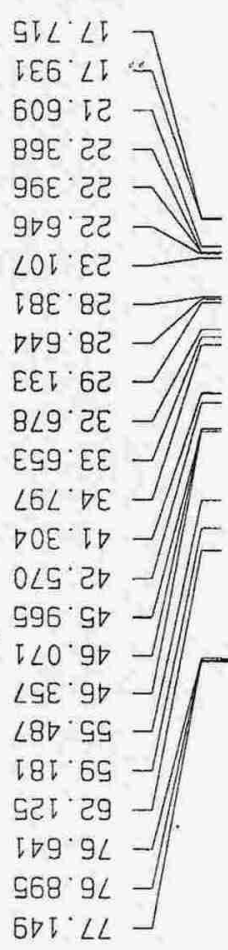

$\angle 6 I^{\circ}$ II

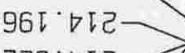

टटE $\nabla I 2$

टLS $\nabla L Z$
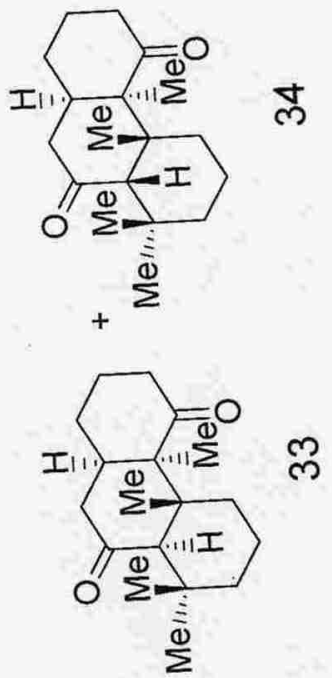